Des Choses, des gestes, des mots

\title{
Une soupe peu ordinaire
}

Analyse du repas des funérailles chez les Mongols

An Unordinary Soup

\section{Sandrine Ruhlmann}

\section{(2) OpenEdition}

\section{Journals}

Édition électronique

URL : https://journals.openedition.org/tc/4688

DOI : $10.4000 /$ tc. 4688

ISSN : 1952-420X

Éditeur

Éditions de l'EHESS

Édition imprimée

Date de publication : 15 août 2009

ISBN : 978-2-7351-1235-7

ISSN : 0248-6016

Référence électronique

Sandrine Ruhlmann, « Une soupe peu ordinaire », Techniques \& Culture [En ligne], 51 | 2009, mis en ligne le 15 juin 2011, consulté le 29 septembre 2022. URL : http://journals.openedition.org/tc/4688 DOI : https://doi.org/10.4000/tc.4688 


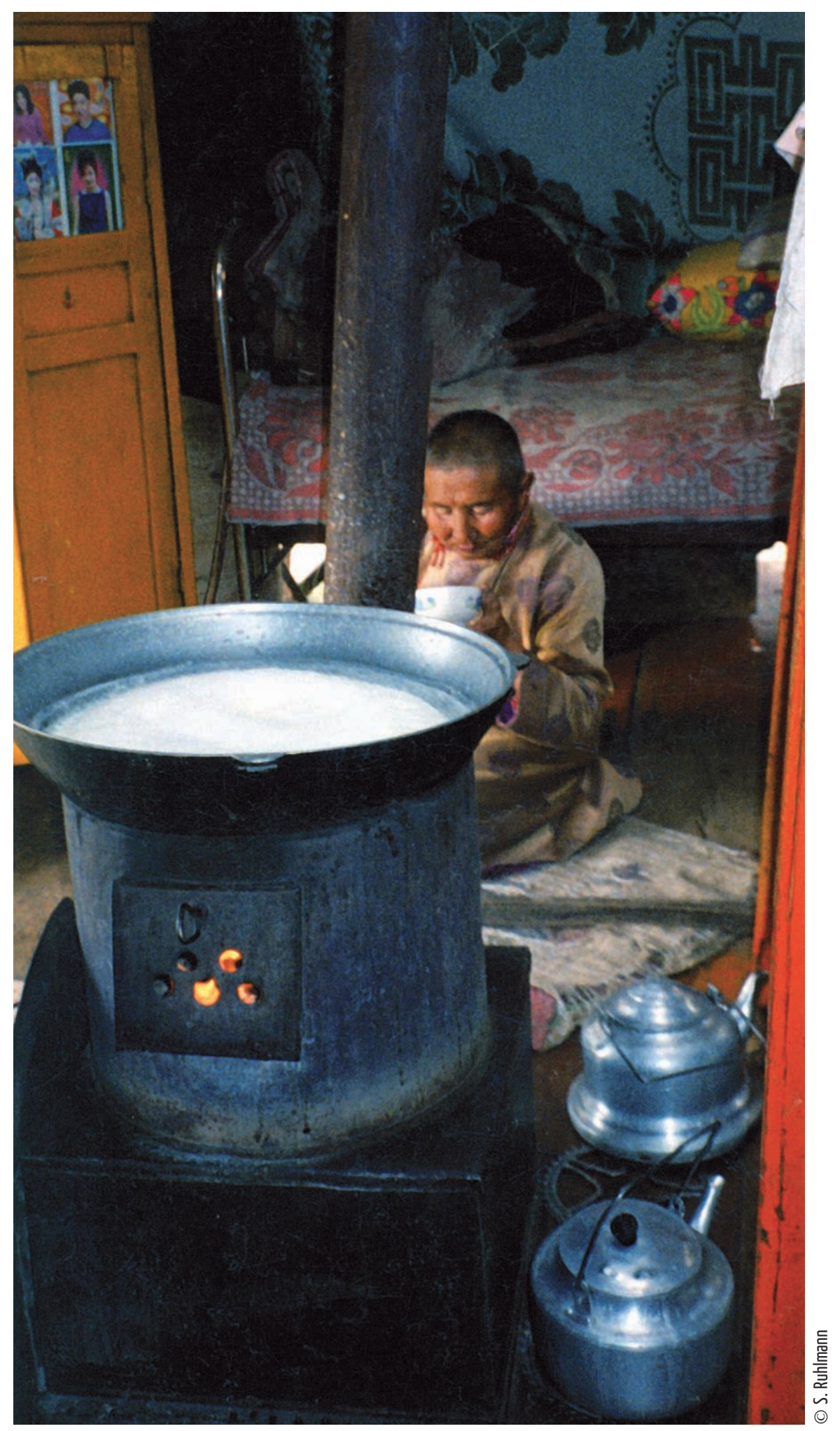




\section{UNE SOUPE PEU ORDINAIRE}

\section{Analyse du repas des funérailles chez les Mongols}

L'auteur montre comment une série d'inversions, que justifie la mort d'un humain, se retrouve jusque dans les techniques de préparation, de distribution et de consommation de la viande de la soupe offerte au repas des funérailles - soupe revêtant une forme toute particulière.

En anthropologie, l'étude des rituels funéraires est une source inépuisable et, dans ce contexte, l'analyse des repas funéraires et des offrandes aux morts, aux ancêtres et aux divinités est particulièrement pertinente. Ainsi, dans son livre sur le système culinaire des Jaina en Inde, Marie-Claude Mahias (1985) consacre un sous-chapitre aux « nourritures particulières » relatives à la mort, tandis que Nur Yalman (1971) s'intéresse aux offrandes alimentaires à Ceylan, dont celles, spécifiques, destinées aux entités surnaturelles et Claudine Vassas (2001), pour la Roumanie, livre un article détaillé sur les modalités de confection, de distribution et de consommation des nourritures « pour le mort » et analyse les partages de ces nourritures entre les vivants et le mort. Si Roberte Hamayon (1990), Caroline Humphrey (1999) et Marie-Dominique Even (1999) se sont intéressées à la question de la mort, de l'âme et du rituel funéraire chez les peuples mongols, elles n’ont pas observé ni analysé les repas funéraires. Or, en République de Mongolie ${ }^{1}$, comme dans d'autres sociétés de culture bouddhique, le partage des nourritures avec et pour le mort est essentiel. Si le partage est fondamental, pourquoi, dans ce cas, les Mongols offrent-ils à l'occasion d'un 
événement important une soupe qui, dans le contexte habituel du partage et de la consommation de la nourriture, offenserait toute personne à qui on l'offrirait? À partir d'un cas particulier qu'il nous a été donné d'étudier², nous montrerons comment ce phénomène d'inversion sous-tend dans les faits, y compris techniques, de la préparation de la soupe, la dynamique historique, politique et religieuse, qui a permis aux Mongols de réajuster leurs pratiques funéraires chamaniques pour les faire coïncider avec les exigences religieuses du clergé bouddhique, puis les lois antireligieuses du gouvernement communiste, et par là même de maintenir la coutume du nourrissement de l'âme du mort.

\section{La conception mongole du repas}

En Mongolie, société d'éleveurs nomades, le modèle du repas n'a pas pour centre ou aliment de base un farineux, comme c'est le cas dans les cultures agricoles. La Mongolie fait partie des exceptions qui n'entrent pas dans le schéma théorique de Sidney W. Mintz (1985, 2001), car même si aujourd'hui l'alimentation ne repose plus uniquement sur les produits issus de l'élevage, la viande et les laitages demeurent les aliments qui nourrissent.

Les Mongols consomment trois repas par jour et, à l'exception du petitdéjeuner qui ne se compose que d'un bol de thé dans lequel on fait éventuellement tremper les restes de la veille, tout repas se compose d'un plat, la soupe, et d'une boisson, le thé. L'un et l'autre sont successivement servis dans le même bol, dans un ordre de présentation et de consommation immuable: thé, soupe, thé.

\section{La soupe: préparation, distribution, consommation}

En Mongolie, la soupe (šöl) est par définition une soupe de viande, c'est pourquoi l'expression pour la désigner ne comporte pas le terme mah exprimant la viande ${ }^{3}$. Pour un Mongol, la viande est l'aliment qui nourrit et fait repas ${ }^{4}$; elle permet de s'accomplir en tant que Mongol et, de nos jours encore, un jeune enfant devient un être social dès lors qu'il consomme de la viande sur l'os appelée « os » (jas) (Fig. 1) ${ }^{5}$. Caractéristique de la considération mongole de la viande et de la soupe, l'expression « soupe noire » (har šöl) désigne une soupe sans adjonction d'autre aliment que la viande elle s'emploie pour la distinguer d'une soupe à laquelle on a mêlé du lait, qui, à l'opposé, est qualifiée de blanche.

La viande se consomme de préférence fraîche et grasse, détachée de l'os ou attachée à l'os qui, suivant l'usage mongol, donne son nom à la part de viande quand celle-ci est consommée sur l'os - les Mongols ne mangent pas du gigot, mais du fémur. Pour la soupe, la viande est généralement prélevée d'un fémur de mouton, mais une fois détachée de l'os, la viande 
n'est autre que des morceaux de chairs dont on ne peut plus identifier la provenance. Installée au sud-est de la yourte, partie dévalorisée de celle-ci, réservée aux femmes et consacrée aux opérations de cuisine, la maîtresse de maison sépare la chair de l'os à l'aide d'un hachoir. Sur une planche en bois, posée sur le sol, un tabouret ou un lit, la chair est découpée au hachoir en morceaux de même taille, suffisamment petits pour qu'ils ne nécessitent aucune découpe au moment de la consommation ${ }^{7}$. Des petits morceaux de gras durs sont découpés à part et ajoutés aux morceaux de chair grasse.

On fait revenir la viande dans le fond de la marmite en fonte, avec un oignon préalablement haché menu et saisi sans matière grasse. Dès qu'elle brunit à l'extérieur, on verse de l'eau pour faire bouillir les morceaux de chair et de gras. En Mongolie, le bouilli est le mode de cuisson privilé-

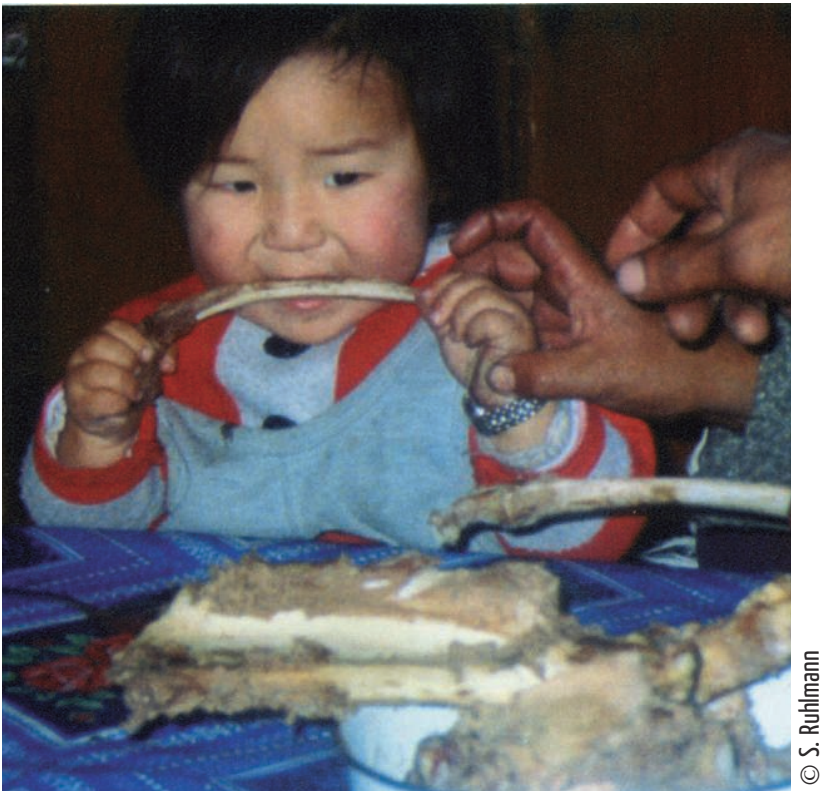
gié de tous les aliments. Il est le mode de cuisson

d'excellence pour la viande, parce qu'il permet de préserver les morceaux de gras dur (ööh) et le gras dur de la chair. L'eau de cuisson retient également toute la graisse (tos) et les sucs qui se dégagent de la chair. On laisse mijoter jusqu'à ce que la viande devienne gris-brun à l'intérieur. Préparées en fin de journée, des pâtes plates et courtes de confection maison ${ }^{8}$ peuvent être ajoutées, pour donner de la consistance à la soupe, qu'on agrémente, en ville, de légumes découpés en morceaux de même taille que la viande.

Le bouillon est communément désigné par le terme šöl, « soupe », mais il peut également être désigné par le terme šüüs, qui désigne dans ce cas précis le jus gras qui s'écoule de la viande à la cuisson, ce même jus gras des raviolis bouillis que l'on aspire en les consommant, la graisse qui coule le long des manches lorsque l'on consomme des parts de viande rattachées à l'os. Le gras symbolise la prospérité du troupeau et pour cela on ne le gaspille pas, ni au moment de la cuisson, ni lors de la consommation.

La soupe est donc composée d'un élément solide, la viande, et d'un élément liquide pour la faire cuire, le bouillon. La viande, fraîche et grasse, a la particularité d'être préparée en morceaux de chair indifférenciés, c'està-dire non identifiables, et d'être distribuée sans distinction de statut des convives, soit de manière égalitaire. Le bouillon, quant à lui, a pour seule caractéristique d'être gras. Il y a une manière précise de consommer la soupe: on mange les morceaux de viande (éventuellement les pâtes et les légumes) en les prélevant à la grosse cuiller, puis on aspire bruyamment le bouillon encore bouillant. La soupe se mange (ide-), tandis que le bouillon se boit (uu-). Le bol, offert et reçu de la main droite, est tenu de la main gauche pour manger, puis de la main droite pour boire. En règle générale, on ne pose pas son bol, on le tient dans la main. Se lever et le poser sur la table basse signifie que l'on a fini son repas.

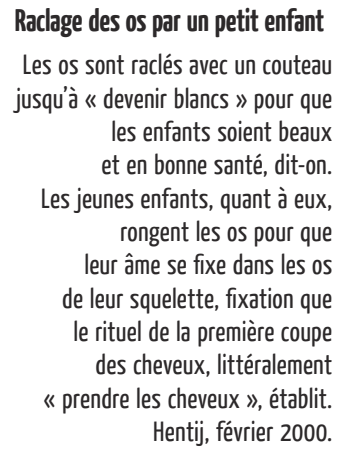

(Fig. 1) 


\section{Le mouton entier bouilli, le plat de viande d'excellence}

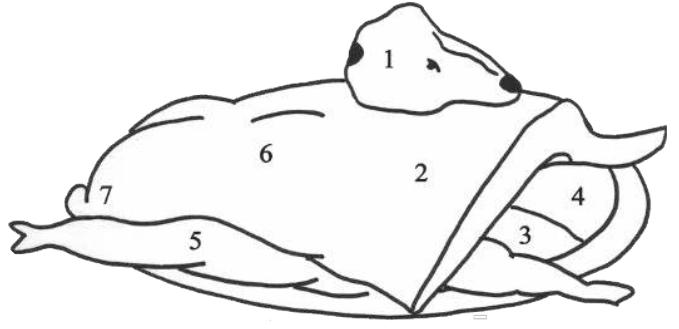

\section{Dessin de šü̈̈s,}

plat de mouton offert au nouvel an

1- la tête (tolgoj)

2- les vertèbres thoraciques (seer)

3- les côtes hautes (öndör havirga)

4- les omoplates (dal)

5- les fémurs (dund čömög)

6 - les vertèbres lombaires (nuruu)

7- le sacrum, avec la queue (uuc, sü̈l)

(Fig. 2)
Auparavant, on ne consommait pas quotidiennement de la viande et celle-ci n'était pas préparée sous forme de soupe. On abattait un mouton, le dépeçait suivant les articulations et l'on faisait bouillir les parts dépecées dans la marmite. Les convives recevaient une part de viande attachée à un os, qui

leur revenait en fonction de leur statut, de leur âge et de leur sexe. La chair était mangée sur l'os ou à l'aide d'un couteau porté à

la bouche et sa consommation s'accompagnait d'un bol du bouillon de cuisson de la viande, nécessairement gras. Le repas se composait donc d'une part de viande fraîche et grasse distinctive, hiérarchique, et d'un bol de bouillon gras.

Pour honorer un visiteur, les Mongols présentaient un plat de mouton bouilli appelé šüüs - ce terme désigne à la fois le mouton abattu pour l'occasion, le plat de viande, le jus gras, et le bouillon gras de cuisson. Le mouton entier, dépecé et bouilli, était présenté sur un grand plat suivant l'ordre usuel des sept parts, chacune attachée à un os $^{9}$. À l'avant du plat, les deux omoplates et les quatre paires de côtes longues ou hautes. À l'arrière du plat, les deux fémurs. Au-dessus de ces parts, le sacrum, la queue et les vertèbres lombaires. Enfin, les vertèbres thoraciques et la part la plus valorisée sociologiquement, la tête, placée sur « le dessus » et constituant « le meilleur » que le même terme, deežz, désigne en mongol ${ }^{10}$ (Fig. 2).

Ce plat honorifique par excellence se prépare encore aujourd'hui pour célébrer les fêtes, une naissance, un mariage, le nouvel an lunaire, et le mode de distribution des parts demeure distinctif - encore aujourd'hui, les meilleures parts sont réservées aux hommes et aux personnes âgées et l'omoplate revient à l'oncle maternel (Hamayon 1975 : 107) (Fig. 3). En aucun cas on ne préparerait ou présenterait ce plat à l'occasion d'un décès.

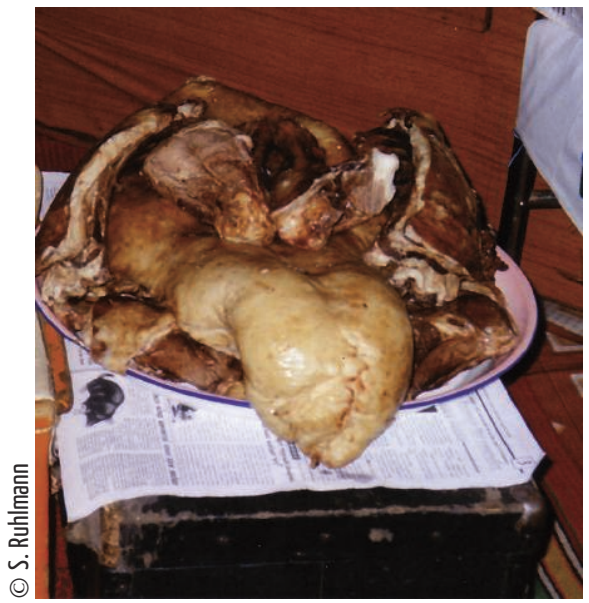

\section{Plat de šï̈́s}

La tête, trop longue à faire bouillir, ne fait souvent plus partie du plat de viande festif. La queue, la partie la plus grasse de l'animal, est alors le morceau d'excellence offert au feu du foyer domestique. Elle pend ici généreusement du plat. Hentij, février 2000

(Fig. 3) 
Voyons de plus près sous quelle forme la viande est présente dans le repas des funérailles chez les Mongols halhs contemporains. Voyons de quelle manière elle est préparée et consommée, pour comprendre pourquoi les familles n'offrent plus un plat de mouton entier bouilli en la circonstance d'un décès, tandis qu'à une haute époque, de la viande était offerte et consommée en quantité à l'occasion des funérailles d'un membre du clan.

\section{La soupe des funérailles}

\section{Techniques de préparation, distribution, consommation}

La soupe des funérailles est composée de divers aliments, que les jeunes parentes de la défunte et du veuf et moi-même avons préparés et versés dans l'eau de cuisson bouillante dans l'ordre suivant:

- des carottes coupées en fines rondelles, des pommes de terre et des citrouilles coupées en dés, de l'oignon et de l'ail hachés menus au hachoir sur planche en bois,

- des pâtes de farine de blé confectionnées sur la base de petits disques de pâte roulés, enduits d'huile et cuits à la vapeur dans un récipient étagé en métal. Les rouleaux sont alors découpés pour obtenir de longs et fins spaghettis (Fig. 4). Ces pâtes diffèrent des pâtes ordinaires: elles ne sont pas bouillies comme le sont les pâtes de la soupe consommée habituellement, mais cuites à la vapeur; elles doivent être plus fines que les spaghettis industriels mongols ${ }^{11}$; de plus, la farine est soigneusement choisie au marché, pour que, une fois cuites, elles ne soient pas brunes, mais blanches,

- des spaghettis (de farine de riz) chinois industriels, d'un blanc translucide,

- et des boulettes de viande dégraissée.

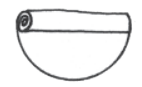

pâton de pâte abaissé

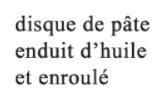

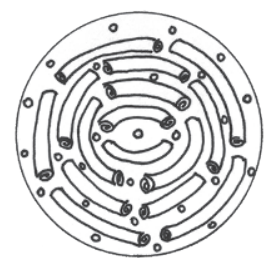

cuisson à la vapeur
des rouleaux de pâte dans le cuit-vapeur étagé

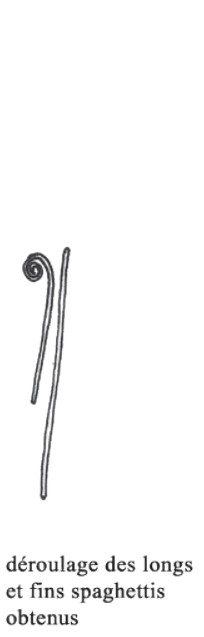

et fins spaghettis obtenus
Spaghettis de funérailles

Séquences techniques de préparation des rouleaux de pâte et de découpe des fins spaghettis de la soupe des funérailles

(Fig. 4)

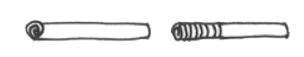

$\begin{array}{ll}\begin{array}{ll}\text { rouleau } \\ \text { cuit }\end{array} & \begin{array}{l}\text { rouleau découpé } \\ \text { en fines rondelles }\end{array}\end{array}$ 


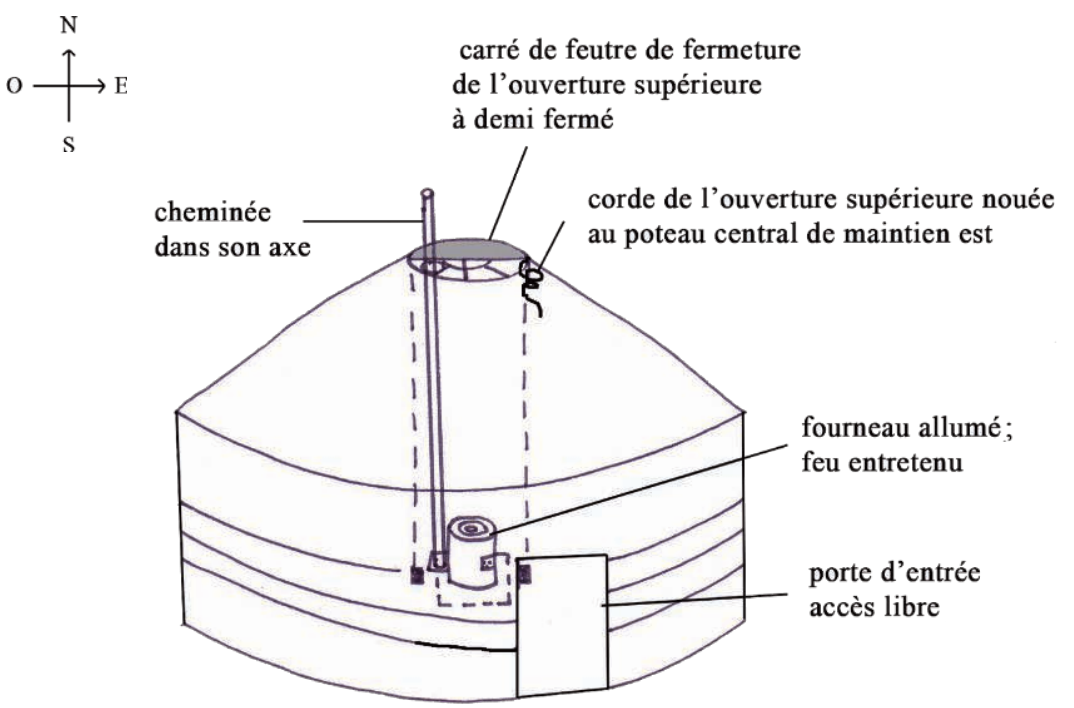

\section{Yourte}

La yourte doit respecter certains codes d'entretien et d'agencement relatifs à son architecture et aux règles sociales de vie:

- le feu est toujours entretenu,

- l'ouverture supérieure permettant l'aération n'est fermée qu'à demi,

- la cheminée est dans son axe,

- la corde reliée à l'ouverture supérieure est nouée au poteau central de maintien de la partie est de la yourte, - enfin, la porte d'entrée n'est jamais condamnée pour permettre à un visiteur d'entrer à tout instant du jour ou de la nuit.

(Fig. 5)

Si la soupe des funérailles est composée d'éléments solides, dont des morceaux de viande, et d'un élément liquide, un bouillon, elle ne possède pas les qualités de la soupe mongole: ses deux éléments de base - viande et bouillon - n'ont pas la propriété d'être nourrissants. Pour être plus précis, la viande de la soupe des funérailles ne correspond pas à la définition mongole de la viande, sous tous ses critères: elle n'est ni découpée en petits morceaux carrés ou rectangulaires, ni grasse, ni bouillie. Elle n'est pas mise à cuire, et elle n'est pas non plus consommée dans un bouillon de cuisson gras. En effet, après avoir séparé la chair fraîche de l'os - un fémur de mouton décongelé, et non abattu spécialement pour l'événement-, nous avons ôté le gras au moyen d'un hachoir (premier dégraissage de la viande). Nous avons ensuite découpé la chair en morceaux et broyé les morceaux, un à un, dans un hachoir à manivelle. Nous avons moulé cette chair ainsi hachée dans le creux de nos mains et modelé des boulettes, que nous avons fait rôtir dans un four électrique (second dégraissage de la viande) ${ }^{12}$. D'une viande à l'origine fraîche et grasse, nous avons façonné des boulettes de chair dégraissée $e^{13}$.

Quant au bouillon de la soupe des funérailles, il est maigre, parce qu'aucune viande grasse n'y a cuit. L'eau de cuisson sert dans le cas présent à faire cuire les spaghettis translucides et les légumes, à faire réchauffer les pâtes précuites à la vapeur, ainsi que les boulettes de viande prérôties, introduites dans l'eau au dernier moment dans le but de les réchauffer avant de servir la soupe. Si la viande de la soupe des funérailles n'est pas comme à l'accoutumée une viande bouillie, qui techniquement bout pendant une vingtaine de minutes, il n'est pas inhabituel de la faire cuire en premier, avant même de verser l'eau de cuisson dans la marmite. Nous avons vu que, de coutume, on fait saisir la viande et nous venons de montrer comment la viande dégraissée de la soupe des funérailles est mise à rôtir. Dans les deux cas, la première opération technique de traitement de la viande 


\section{Yourte mortuaire}

L'agencement de l'espace intérieur de la yourte du défunt est transformé par la crainte de la souillure et de sa propagation possible. La yourte devient une yourte mortuaire : vidée des affaires personnelles, elle renferme le cadavre qui doit être isolé.

La yourte mortuaire est une yourte inversée en tout point :

- le feu n'est plus entretenu,

- l'ouverture supérieure permettant l'aération est entièrement fermée,

- la cheminée est sortie de son axe,

- la corde reliée à l'ouverture supérieure est nouée au poteau central de maintien de la partie ouest de la yourte,

- enfin, l'accès est condamné de l'extérieur par une planche en bois, fixée latéralement par des cordes à la corde de fixation des treillis du milieu et au chambranle de la porte.

Au quotidien, habiter une telle yourte reviendrait à refuser toute hospitalité, comportement en contradiction avec la culture mongole nomade. Mais en contexte funéraire, ces aménagements au sein de l'habitat protègent les vivants de la souillure et permettent à la famille d'entamer le processus de séparation d'avec le défunt.

(Fig. 6)

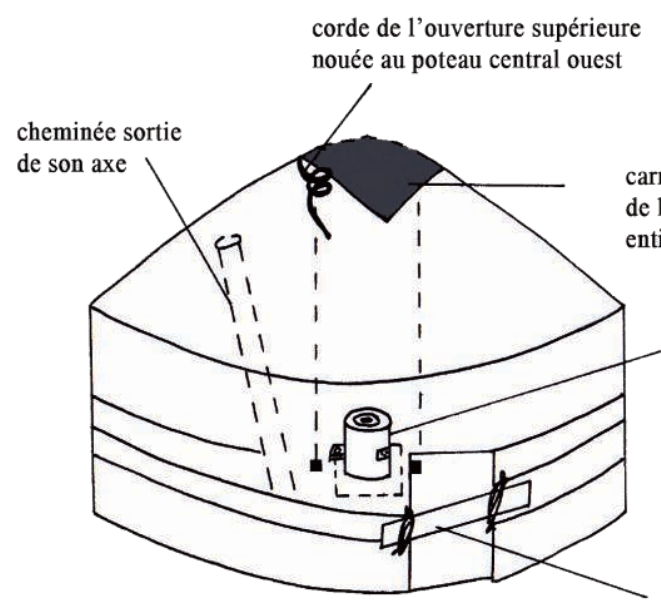

carré de feutre de fermeture de l'ouverture supérieure entièrement rabattu

fourneau éteint; feu pas (plus) entretenu

planche de condamnation de la porte d'entrée, ficelée aux chambranles

est donc quelque peu similaire (saisie ou rôtie), mais les propriétés de la viande n'étant pas les mêmes (grasse/dégraissée), le résultat est différent: une viande est bouillie et grasse, l'autre rôtie et dégraissée; par extension, une soupe est grasse, l'autre maigre.

Malgré tout, la soupe des funérailles est distribuée et consommée de la manière habituelle: les hommes servis en premier, les femmes en dernier, chacun mangeant les aliments de la soupe avant d'en boire le bouillon.

\section{Une soupe inversée}

Chez les Mongols, le cadavre constitue une souillure majeure contagieuse. La famille en deuil doit s'en protéger par de multiples inversions, dont le rituel funéraire est ponctué: la toilette du mort, l'aménagement de la yourte du défunt où il est isolé14 (Fig. 5 et 6) jusqu'à son enterrement, la mise en bière, les habits des membres du cortège funéraire, la sortie du cadavre de la yourte ${ }^{15}$. Ces inversions de gestes, de mouvements, de tenue des objets, de port des habits, de sens de circulation, protègent de la souillure et du malheur qui découlerait de la contagion, parce qu'elles entraînent un renversement des valeurs qui s'expriment habituellement dans les actes. Elles permettent en outre à la famille d'entamer le processus de séparation avec le défunt.

Des inversions se retrouvent jusque dans l'alimentation, y compris dans les opérations techniques de la préparation de la soupe. La soupe des funérailles est appelée « soupe de légumes et de pâtes » (nogootaj guriltaj šöl), et non pas « soupe » (šöl) comme à l'accoutumée, ce qui ne suffit pas à désigner les boulettes amaigries comme n'étant pas de la viande, puisque pour la soupe qui comporte toujours de la viande, cette dernière n'est jamais expressément nommée. La viande de la soupe funéraire est une 


\section{Sacrifice de cheval chez les Bouriates au début du XXe siècle}

Les photos nous montrent les participants rassemblés pour le partage de la viande sacrificielle, ainsi que le sacrifice d'un cheval, maintenu sur le dos, les pattes avant et arrière liées deux par deux et tenues par des sacrifiants, pour que le sacrificateur opère vite et de manière efficace. (Curtin 1909 : 44-46)

(Fig. 7, 8, 9 et 10) antithèse de la viande telle qu'elle est consommée par les Mongols, telle qu'ils la font exister socialement et lui confèrent une valeur honorifique. Ainsi, les femmes qualifient les boulettes, non pas de viande maigre dite « viande noire » (har mah), mais de « viande sans gras » (ööhgüj mah). C’est significatif puisque, grasse par définition, on ne précise communément pas que la viande est « avec du gras» (öötej). Elles affirment clairement que « ce n'est pas de la viande! » (mah biš!). La soupe des funérailles est une soupe inversée et, à ce propos, le mode de préparation (dégraissage) et de cuisson (rôti) des boulettes constitue une double inversion de la viande telle qu'elle est consommée au quotidien (grasse et bouillie). Nous rejoignons là des bases élémentaires du triangle culinaire de Claude Lévi-Strauss (1965, 1967 : 390-411), puisqu'en Mongolie, le rôti est l'affaire des hommes, quand ils chassent le gibier dans la nature sauvage, il s'effectue donc à l'extérieur de la maison, avec le minimum d'ustensiles de cuisine (Ruhlmann 2006 : 398, Hamayon 1975 : 109). À l'inverse, le bouilli est attribué aux femmes, qui cuisinent la viande du bétail à l'intérieur du foyer domestique, avec tous les ustensiles de cuisine nécessaires. Dans le cas de la Mongolie, l'opposition n'est pas tant celle de la nature et de la culture, que celle de la mort et de la vie. La viande de la soupe des funérailles est à la fois une inversion de la viande réservée aux repas quotidiens et une inversion de la viande réservée aux repas festifs, qu'elle soit consommée sous forme de soupe ou non, puisque, dans les deux cas, elle est obligatoirement grasse. Néfaste, la mort ne saurait être associée à la fête et perturbe le quotidien, au sens où elle trouble négativement le cours normal de la vie des vivants, tout en étant cachée aux mauvais esprits particulièrement craints. Si les vivants sont en deuil, les mauvais esprits ne doivent surtout pas en avoir connaissance. L'interdit de prononcer les mots «mort » (ühel), « cadavre » (hüür), « dépouille » (šaril), qui évoquent la mort, permet de cacher l'existence d'un cadavre et de tromper les esprits affamés, qui peuvent s'attaquer aussi bien au corps du mort qu'à son âme.

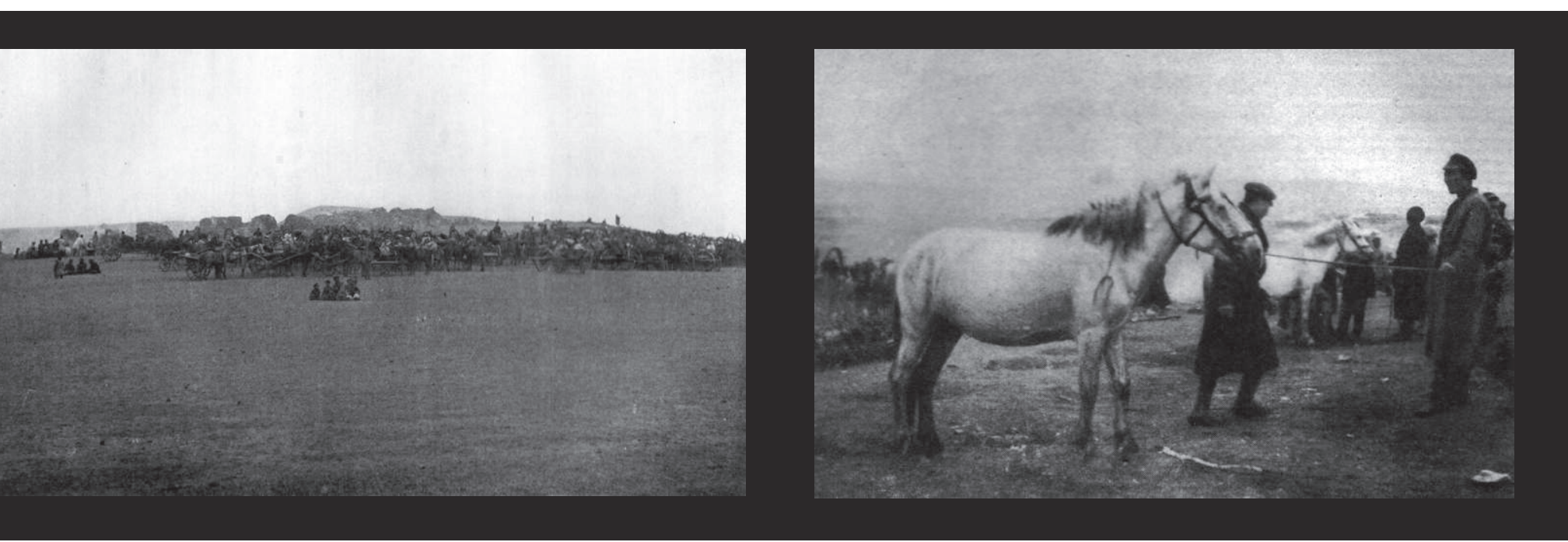


La soupe du repas des funérailles est préparée et offerte pour faire barrière à la souillure que représente le cadavre. Pour cela, les qualités des composantes de la soupe sont inversées (viande et bouillon non gras) et les valeurs qui leur sont attachées sont renversées (viande et bouillon socialement dévalorisés). Il n'est pas surprenant qu'un événement extraordinaire comme la mort induise une préparation et une consommation inhabituelles, voire exceptionnelles. Comment expliquer cependant que les Mongols préparent et consomment, à l'occasion des funérailles, des boulettes de viande dont ils disent que ce n'est pas de la viande? Pourquoi dans ce cas ne pas choisir de faire abstinence de viande? Un retour dans l'histoire sur sept siècles va nous permettre de comprendre ce choix.

\section{La viande de sacrifice clanique chez les Mongols médiévaux}

Au XIII siècle, chez les Mongols, le sacrifice et l'offrande étaient liés et le terme tahilga désignait l'ensemble des offrandes, oblations et sacrifices destinés aux mânes des ancêtres ${ }^{16}$ ou aux esprits territoriaux. À l'occasion d'un décès, l'offrande sacrificielle consistait en un « sacrifice de nourriture brûlée » (tüleši) (tülš selon la translittération adoptée du halh moderne). L'offrande était composée essentiellement d'une viande. La «part de l'offrande [destinée] aux ancêtres » (kešig) [hešig] était distribuée aux assistants sous la forme de « restes de la viande sacrifiée » (bilevür), ainsi que de « restes de la boisson [spiritueuse] de sacrifice » (sarqud) ${ }^{17}$ (Ligeti 1973).

Dans l'Histoire secrète des Mongols, une réplique d'hommes rejetés du clan est explicite au sujet du sacrifice de viande offert aux ancêtres: « Comment osez-vous nous faire arriver en retard à [la distribution de] la viande et [de] l'alcool provenant de la part des ancêtres? » (Even \& Pop 1994 : 57).

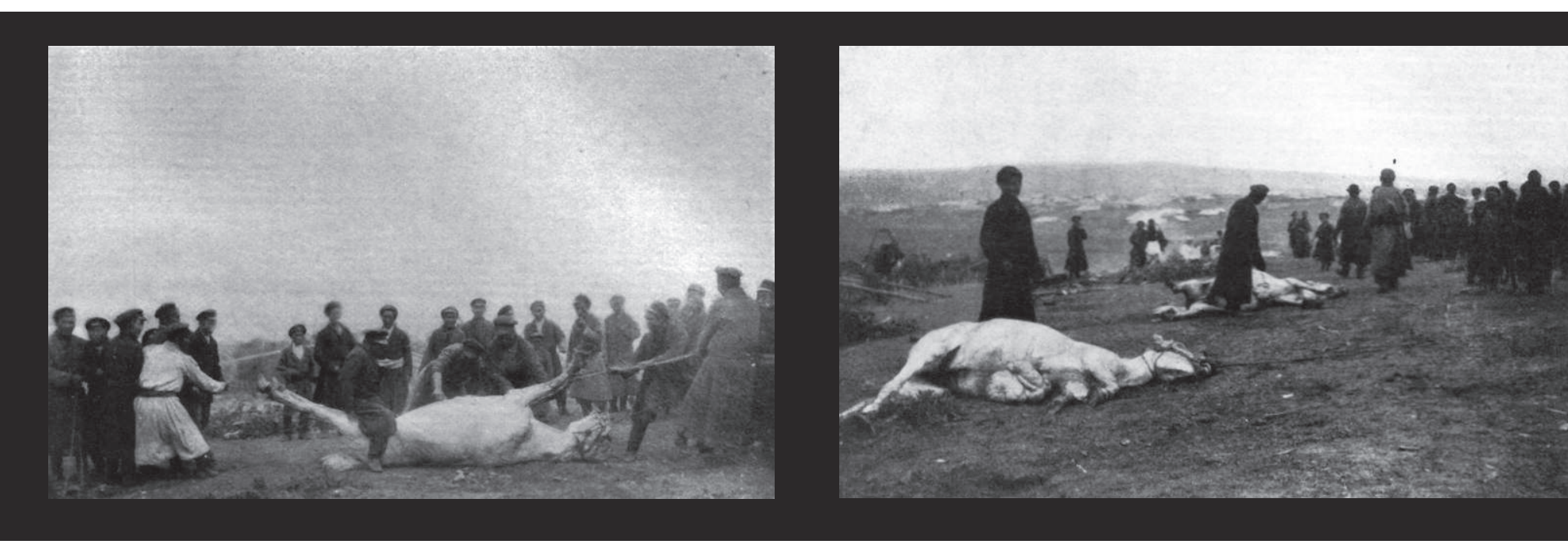


Le terme kesig [hešig] « part » désigne la part de viande sacrificielle offerte aux ancêtres: une petite part destinée aux mânes des ancêtres était brûlée dans le feu; une autre part devenue, par le rituel, porteuse de la « grâce [ancestrale] » (kesig [hešig]), était ensuite distribuée à tous les descendants. La part distribuée et consommée permettait de tirer bénéfice des solidarités inhérentes au clan. Être exclu de la distribution/consommation de cette part signifiait être exclu du clan, tout au moins relégué à ses marges.

Les sources dont nous disposons concernent les sacrifices des Mongols de Sibérie (Fig. 7 à 10). Les auteurs décrivent par étapes l'organisation et le déroulement des rituels, qui sont identiques, à l'exception des dédicataires qui en constitueraient la seule variation. Ainsi, chez les Bouriates, on collectait auprès des participants tout le nécessaire pour le rituel, dont le(s) animau(x) sacrifié(s), l'alcool de lait pour les libations et le bois pour la viande sacrificielle. Les participants s'installaient, par lignage et par famille, à flanc de montagne, devant une marmite pouvant contenir un animal entier. Chaque animal sacrificiel recevait une libation de lait de jument fermenté. Une fois l'animal abattu, dépouillé et débité, la viande cuisait plusieurs heures, pendant lesquelles les participants festoyaient (Hamayon 1990 : 637-643, Curtin 1909 : 44-52).
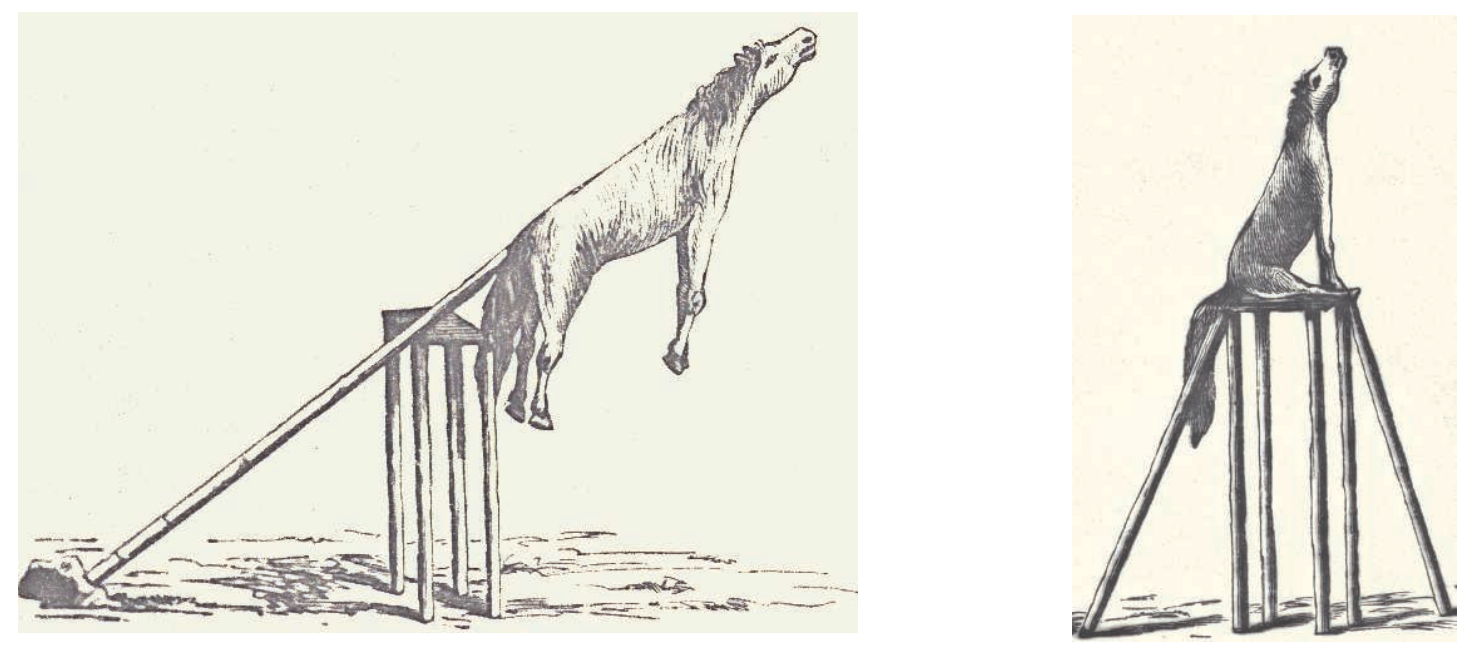

\section{Constructions sacrificielles en bois}

Les dépouilles des animaux sacrifiés étaient enfilées sur des perches en bois fixées sur des installations inclinées en direction des dédicataires du sacrifice. Construction sacrificielle des Altä̈ens (Radlov 1893, T.2, p.18) et des Téléoutes (Radlov 1893, T.1, p.332). (Fig. 11 et 12)
Chez les Bouriates, peuple de chasseurs, on distinguait clairement la part individuelle (xubi [huv']) égalitaire (chair bouillie, détachée des os) de la viande sacrificielle dite dalanga, porteuse de chance et de la grâce ancestrale (xešeg [hešig]), rapportée par les participants de chaque groupe lignager à la maison, de la part hiérarchique (attachée à l'os), šuladahan, consommée sur le lieu du sacrifice collectif (tajlgan) (Hamayon 1990 : 786 note 19). Chez les Halhs, peuple d'éleveurs, on pouvait distinguer une part de viande sacrificielle grillée offerte aux mânes des ancêtres par l'intermédiaire du feu, ainsi qu’une part de viande bouillie, détachée des os, distribuée en parts égalitaires entre les participants ${ }^{18}$ (Even 1999 : 175). 
Le repas se consommait sur le lieu du sacrifice. Les os de l'animal sacrifié étaient brûlés (Plan Carpin 1965 : 37) et sa dépouille était enfilée sur une perche inclinée, pour garantir le transfert de l'âme de l'animal aux destinataires du sacrifice, les ancêtres, et empêcher sa renaissance dans son espèce sur terre.

Cette distinction entre part rôtie/part bouillie n'existe plus aujourd'hui et il est difficile d'affirmer avec exactitude si les boulettes de viande rôties, consommées par les visiteurs, sont indirectement destinées ou non à l'âme du mort et/ou aux mânes des ancêtres. Toujours est-il que le rôti, le grillé, et plus précisément le gras et la chair brûlés, constituent encore aujourd'hui, chez les Mongols, une offrande pour nourrir les ancêtres, comme les bribes de gras et de chair jetées au feu quotidiennement, avant de servir le repas ${ }^{19}$. Les ancêtres sont, dit-on, friands de brûlé: de viande brûlée,

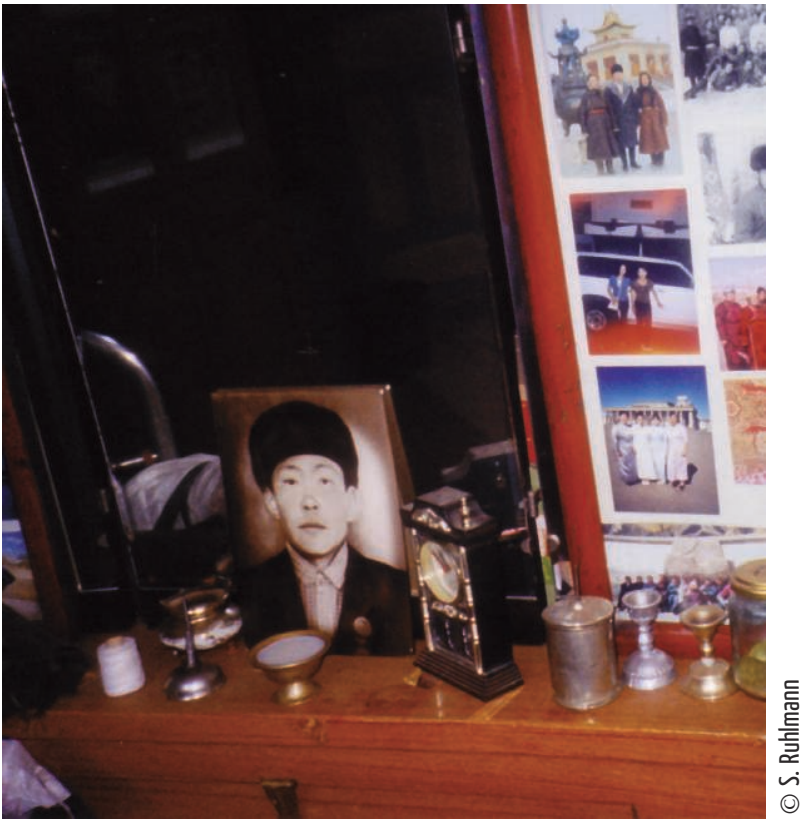
mais aussi de l'odeur qui se dégage du gras et de la chair brûlés. Ainsi, chez les Mongols Ordos, le verbe tüleh signifiant « brûler » sert à désigner une offrande de viande brûlée destinée aux morts, notamment dans l'expression « brûler des mets en offrande » (henšüü dojon tüleh). Le terme henšüü désigne le relent de friture, l'odeur de graisse d'os ou de viande brûlée, le graillon et encore le goût de brûlé; le terme dojon se compose du tibétain du-ba, dud-pa « fumée » et de yon « offrande ${ }^{20}$. Le terme tüleš désigne les mets brûlés sur les tombeaux en offrande aux mânes des ancêtres. Les mets brûlés sont les mets des morts; il n'est donc pas impossible que les boulettes de viande rôties des Mongols halhs soient destinées à nourrir l'âme du défunt. Mais pourquoi ne pas en offrir directement au mort, par l'intermédiaire de son portrait photographique placé sur l'autel funéraire (Fig. 13), comme le sont d'autres nourritures du repas des funérailles, ainsi que nous le verrons?

\section{L'interdit bouddhique de sacrifice animal}

Au XIII siècle, le corps d'un homme de rang supérieur était placé dans un cercueil et l'ancienne règle (zasag) exigeait d'offrir à son âme, sur la tombe, des nourritures provenant d'animaux domestiques sacrifiés. La manière mongole d'abattre le bétail pour la consommation quotidienne ou pour l'offrir en sacrifice consistait alors, ainsi que le grand pouvoir (ih zasag) ${ }^{21}$ le préconisait, à ne pas faire couler le sang hors du corps de l'animal et à ne pas le faire se répandre sur la terre. Pour se faire, on lui « pressait le cœur », selon les termes de V.A. Riazanovski (1965 : 83), qui a étudié les 

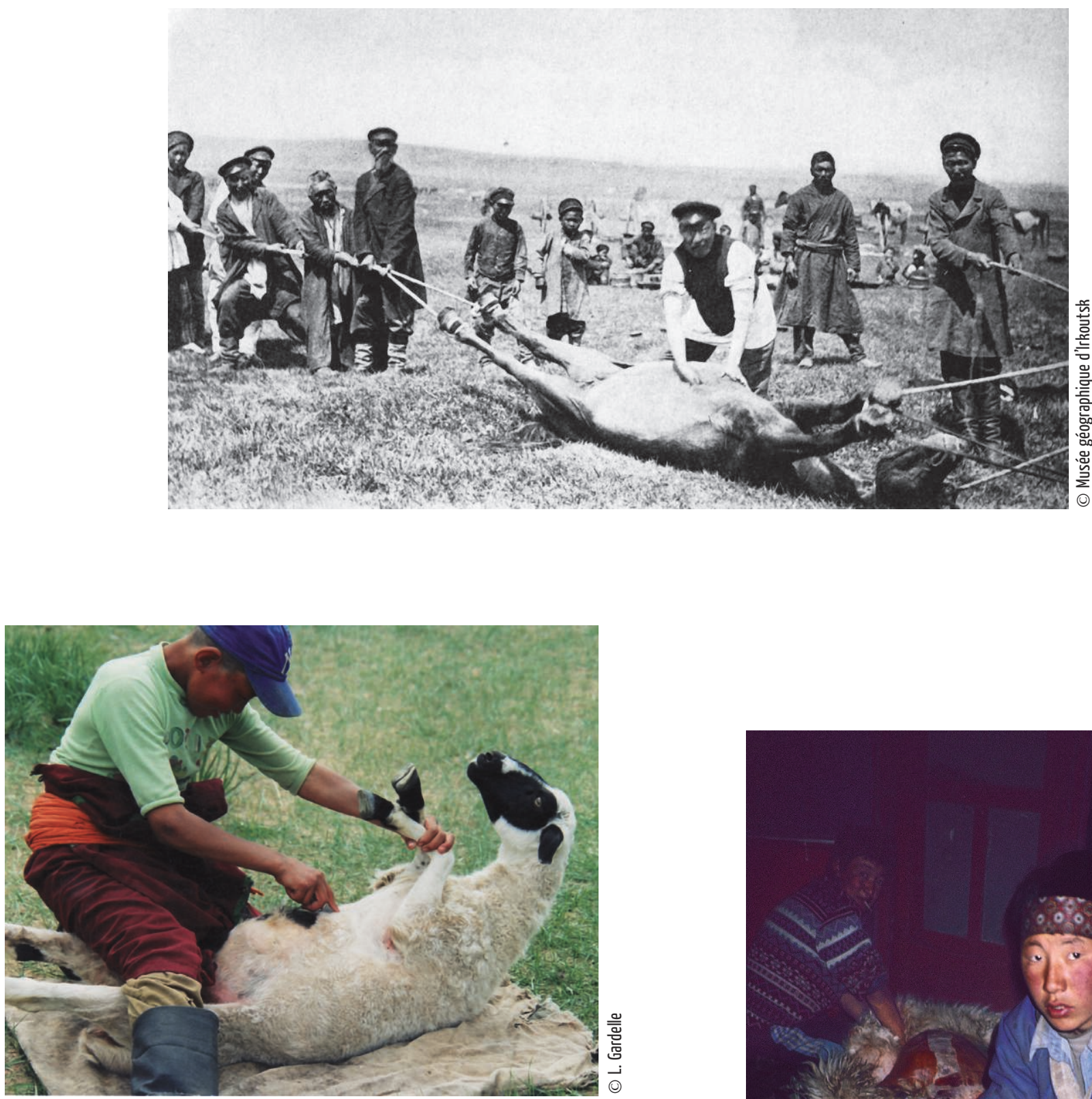

\section{Techniques d'abattage}

Comparaison des techniques d'abattage des animaux sacrifiés et des animaux abattus pour la consommation quotidienne. Les figures 14 et 15 illustrent parfaitement notre propos: l'animal, sacrifié ou abattu (cheval ou mouton), est maintenu sur le dos (par un ou plusieurs hommes) et l'ouverture est pratiquée sous la paire de côtes flottantes.

Fig. 14: Sacrifice de cheval chez les Bouriates. Carte postale éditée par le Musée géographique d'Irkoutsk, ayant pour titre: « Tä̈lgane ». La cérémonie d'oblation chez les Bouriates chamanistes, photo de L.A. Venjukov.

Fig. 15: Abattage d'un mouton chez les Mongols halhs. Arhangaj, juin 1999

Fig. 16: Dépouillage précédant le débitage de l'animal. Bajanhongor, novembre 1998.

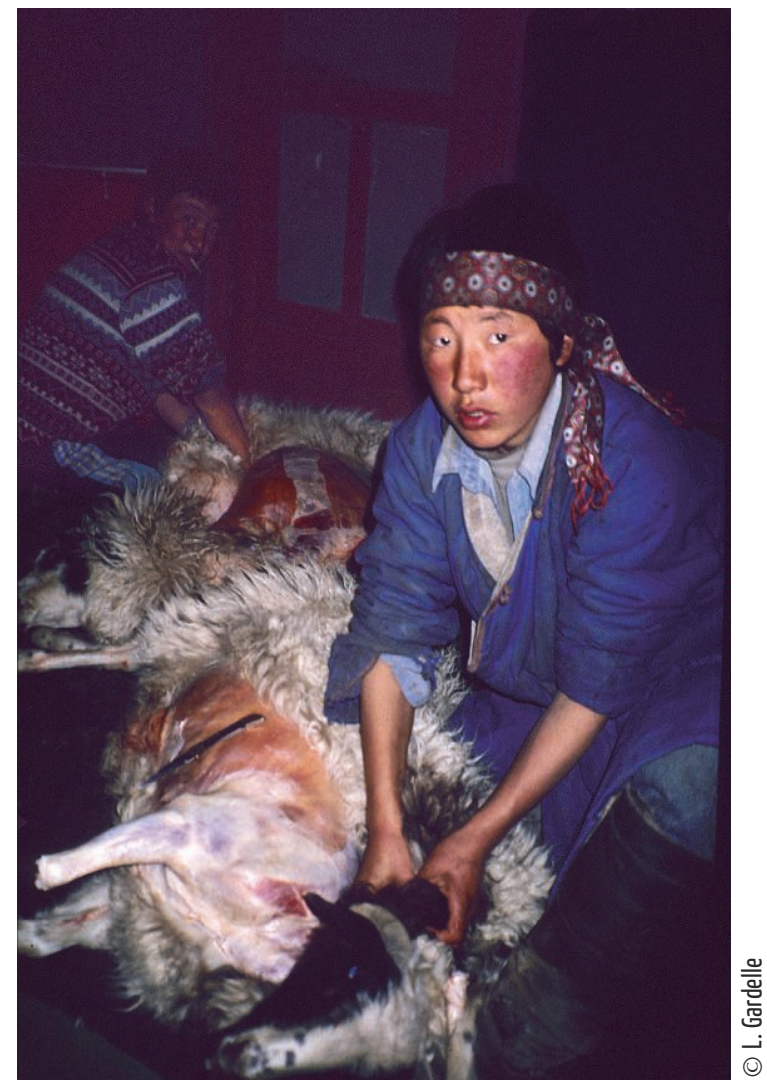


évolutions du code de lois mongol. J'ai observé les techniques d'abattage du mouton; elles n'ont manifestement pas changé. Les membres de l'animal sont liés deux par deux, à l'avant et à l'arrière, pour le maintenir sur le dos. L'abatteur doit alors ouvrir le ventre à l'endroit où se termine le sternum, juste sous la jointure des deux dernières côtes flottantes, passer la main dans l'ouverture, trouver l'aorte thoracique au milieu de la graisse et la presser pour étouffer l'animal en quelques secondes. Il doit ensuite rompre l'aorte thoracique d'un geste sec avec un couteau, ou à main nue d'un geste ferme. Il maintient l'aorte thoracique pour que le sang reste contenu dans la cavité thoracique et ne coule pas sur la terre (gazar), associée au territoire des ancêtres par les personnes âgées. Le sang est recueilli dans un récipient et réservé pour la préparation des boudins par les femmes, tandis que l'animal est dépouillé et débité suivant les articulations par l'abatteur, un homme. Les os sont gardés intacts, pour permettre à l'âme de l'animal de renaître et de perpétuer son espèce. Les parts de viande attachées aux os sont prêtes pour la cuisson (Fig. 14 à 16)

En 1577, alors que l'empereur Altan se convertit au bouddhisme $e^{22}$, le clergé bouddhique promulgue un interdit de sacrifice animal en association à la mort d'un être humain ${ }^{23}$. La spécificité mongole du sacrifice animal résidant dans ce qu'il n'est par définition pas sanglant, tandis qu'il l'est par essence dans de nombreuses sociétés, l'interdit religieux ne concerne pas l'acte de sacrifier en lui-même. Si l'interdit ne le stipule pas explicitement, nous savons que c'est en fait l'offrande de viande animale aux ancêtres qui fut proscrite. Le clergé refusait que des offrandes de viande soient destinées à de simples morts humains; il exigea que le bétail soit désormais consacré vivant aux divinités bouddhiques (Riazanovski 1965 : 114, 190)24.

Après soixante-dix années de gouvernance communiste, précisément depuis une loi antireligieuse et hygiéniste datant de 1956, les Mongols ont l'obligation d'enterrer les morts sous terre, sans distinction de statut, dans des cercueils en bois ${ }^{25}$. Parallèlement, depuis 1990, le pays s'est engagé dans la voie de la démocratisation et de la tolérance religieuse: le bouddhisme est déclaré religion nationale et des formes de néo-chamanismes émergent. Ce nouveau climat a des répercussions sur les pratiques et les croyances des Mongols, notamment sur les pratiques funéraires qui se recomposent au sein des foyers domestiques. Face à cette situation, craignant à la fois que les croyances chamaniques et l'organisation sociale clanique fondée sur les ancêtres émergent à nouveau et que le chamanisme reprenne ses droits sur le bouddhisme, le clergé a dû réagir: il proscrit désormais explicitement, la consommation de viande en association avec la mort d'un humain. Initialement, le clergé avait proscrit le sacrifice animal à l'occasion des funérailles pour supprimer la part de l'offrande sacrificielle destinée aux ancêtres (hešig), garants du bonheur de leurs descendants. Aujourd'hui, il est dans l'obligation d'interdire non plus le sacrifice animal, qui ne se pratique plus, mais la consommation de viande en contexte funéraire, ainsi qu'une technique de préparation et de cuisson de la viande destinée aux ancêtres (la viande rôtie et l'odeur de brûlé). 
Repas des funérailles

(Fig. 17)
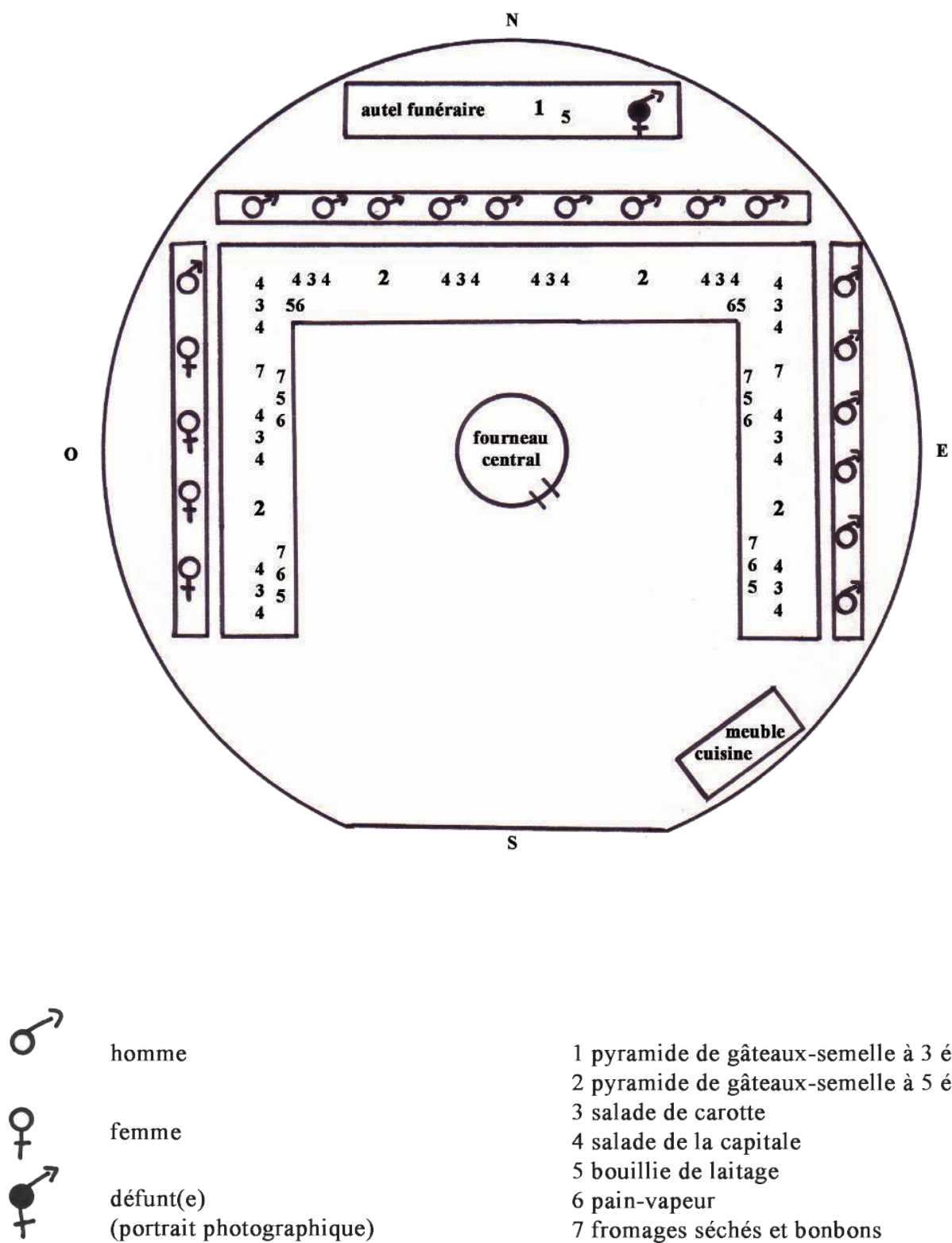

1 pyramide de gâteaux-semelle à 3 étages

2 pyramide de gâteaux-semelle à 5 étages

3 salade de carotte

4 salade de la capitale

5 bouillie de laitage

6 pain-vapeur

7 fromages séchés et bonbons 
Il n'en reste pas moins qu'à l'occasion des funérailles, une soupe contenant de la viande est consommée. Ce sont certes des boulettes de viande rôties qui « ne sont pas de la viande », mais les familles prennent la peine de les préparer suivant des techniques précises, qui évoquent d'anciennes pratiques chamaniques, pour les consommer le jour de l'enterrement. La présence des boulettes rôties dans la soupe funéraire confirme bien que ce n'est pas le sacrifice qui compte pour les Mongols, mais la viande. Les boulettes ont cependant la particularité d'être successivement rôties et bouillies - même si elles ne cuisent pas dans l'eau, elles y sont immergées un temps -, autrement dit leurs modes de cuisson sont successivement ceux, d'antan, de la part des ancêtres (rôtie) et de la part revenant aux participants (bouillie). La consommation des boulettes de viande est en quelque sorte une manière de contourner l'interdit bouddhique, tout en le respectant: préparer une viande qui ne provient pas d'un sacrifice animal et manger de la viande qui n'en est pas. Les boulettes de viande apparaissent alors comme étant une négation de la présence de viande dans la soupe funéraire. Une non-viande serait en fait présente au titre d'une absence de viande exigée par le clergé bouddhique, mais au titre d'une présence exigée par les croyances chamaniques. Afin de mieux comprendre les motivations des Mongols, il est nécessaire d'étudier de près le repas qui intègre la soupe des funérailles.

\section{Le repas des funérailles}

Le repas des funérailles a lieu idéalement le troisième jour après le décès. Les premiers à le consommer sont les membres du cortège funéraire, à leur retour du cimetière ${ }^{26}$. Une « multitude de gens » (zöndöö hümüüs), amis, voisins, puis parents éloignés et enfin parents proches, prennent place par vagues successives.

Le repas des funérailles se compose, comme tout repas mongol, d'une soupe, en guise de plat central, et de thé, pour commencer et finir le repas, mais une multitude d'autres mets sont également offerts (Fig. 17):

- des petits pains vapeur (Fig. 17 [6]), faits de farine de qualité supérieure et de couleur blanche, trempés dans la soupe,

- des salades de crudités: la salade de la capitale [3], d'origine russe, composée de petits pois, jambon, carottes, navets mélangés dans de la mayonnaise industrielle salée et sucrée ; la salade de carottes [4], combinaison de carottes râpées, de chou finement haché, d'oignons émincés, marinés dans une vinaigrette sucrée,

- des bouillies de laitage et de riz [5], l'une chaude et liquide, l'autre froide, compacte et additionnée de raisins secs,

- des bonbons et des fromages séchés [7], des pyramides à cinq étages de gâteaux en forme de semelle [2] décorées, enrichies, de bonbons et de carrés de sucre, (Fig. 18). 


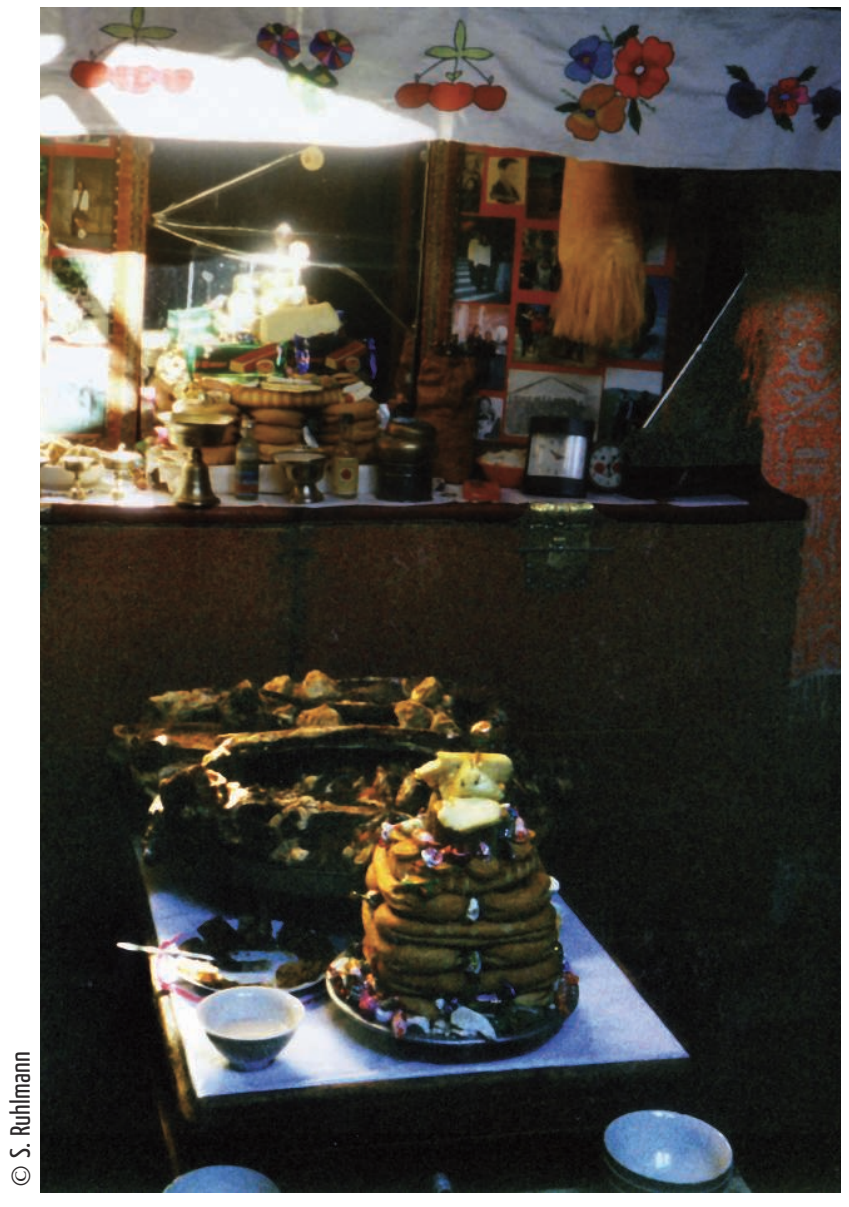

Comme de coutume, le thé et la soupe sont distribués successivement, de la main droite, par des femmes, suivant les règles traditionnelles de distribution: les hommes, placés au nord-ouest, sont servis avant les femmes et les enfants, assis au sud-est, c'est-à-dire les aînés avant les cadets. Par contre, tous les autres plats, uniques ou multiples, sont présentés de manière simultanée sur les tables, suivant une disposition symétrique.

Pour l'ensemble de ces plats, les familles instaurent une sélection parmi les items ordinaires de leur vie alimentaire: le choix porte sur des nourritures qualifiées de « blanches » (cagaan) et dont la mise en valeur dans le cadre du rituel funéraire procède de rites de purification. Ainsi les pains vapeur, les salades, les bouillies de laitages et de céréales, les bonbons et les pâtisseries, de même que la soupe et ses boulettes de viande, sont-ils associés aux produits laitiers dits « aliments blancs ». En leur étant associés, ils s'approprient les qualités des « aliments blancs », qui symbolisent le renouveau, la prospérité des troupeaux ${ }^{27}$. Dans le domaine de la technique, les laitages et les aliments qualifiés de blancs répondent à une notion bouddhique du bonheur: la technique de préparation de chacun des aliments du repas des

Pyramide de gâteaux-semelles du nouvel an lunaire

Cette photographie permet de comprendre comment se présente une pyramide de gâteaux-semelle. Comme ici sur l'autel domestique, une même pyramide est déposée le jour des funérailles devant le portrait photographique du défunt sur l'autel funéraire. Comme ici sur la table basse, les convives consomment le repas des funérailles sur les tables disposées à cet effet. Hentij, février 2000.

(Fig. 18) funérailles consiste soit à les découper en une infinité de petits morceaux de même taille - légumes crus des salades et cuits de la soupe -, soit à les additionner de laitages - le thé, les bouillies, les pâtisseries. Les aliments qui composent chaque plat du repas des funérailles constituent ainsi des «mérites » (bujan), au sens bouddhique du terme. La soupe elle-même est blanchie: habituellement composée uniquement de viande, elle est qualifiée de «noire» (har), mais de multiples morceaux de légumes, pâtes, boulettes s'y ajoutant, elle devient blanche (cagaan). La viande, quant à elle, communément appelée " aliment brun-gris » (bor idee), couleur assimilée au sombre et au noir en Mongolie (Hamayon 1978), est blanchie à partir du moment où elle devient l'inverse d'une viande au sens mongol du terme, soit quand on lui enlève son gras.

Le repas des funérailles est donc composé de "mérites », appelés également « mérites blancs » (cagaan bujan), qui représentent une accumulation de nourritures. Ce n'est pas le volume qui importe, mais la multitude de nourritures, qui repose à la fois sur une grande variété d'aliments (diversité), blancs ou blanchis, et sur la multiplication de chacun d'eux (découpe). Sur le plan culinaire, technique et terminologique, le repas contemporain des funérailles se différencie de l'antique repas sacrificiel funéraire, parce que l'intérêt n'est plus porté sur la ou les parts distinctives ou égalitaires, donc sur la part hešig, mais sur les morceaux nécessairement égalitaires, la multitude de morceaux, soit 
le morceau heseg. Le clergé bouddhique a ainsi instauré un transfert: du sacrifice animal sur les mérites, de la part sur les morceaux, de la viande sur les laitages, du noir sur le blanc.

\section{Les mérites et le blanchiment}

Les techniques de découpe et de cuisson des aliments du repas des funérailles en général, de la viande de la soupe funéraire en particulier, sont au service du principe bouddhique d'accumulation des mérites. Dans la doctrine bouddhique, cette accumulation représente la multiplication des éléments qui est au fondement du principe de vie selon lequel une graine donne une infinité de grains. Ainsi, le jour des funérailles, plutôt que d'ingérer une grande quantité de soupe, on mange une infinité, en variété et en nombre, d'aliments qui la composent. On consomme également de la bouillie de laitage, composée, quant à elle, d'une multitude de grains de riz et de millet.

Au principe d'accumulation des mérites s'ajoute un processus de purification, que la couleur blanche incarne, et qui se matérialise par le biais de pratiques alimentaires d'usage et de consommation d'aliments blancs et/ou mérites. Pendant la cérémonie d'enterrement, la yourte où reposait le cadavre est lavée avec un mélange consacré d'eau et de lait; c'est donc dans une yourte «blanchie » (cajlga-) que le repas des funérailles est consommé. Au cimetière, les membres du cortège funéraire font des offrandes d'une multitude de grains de millet et de riz consacrés sur le cercueil puis sur la tombe, ainsi que des aspersions d'un mélange consacré d'eau et de lait, le même mélange avec lequel ils se lavent les mains et le visage au retour du cimetière. « Tout devient pur et blanc », dit-on. Les verbes cajh, cajlgah et cajrah, « blanchir, rendre blanc ", désignent le blanchiment des objets et des personnes, soit les membres du cortège funéraire, les membres de la famille restreinte et étendue, et les convives, parce que sur leur personne repose un soupçon de contagion par le contact ou la proximité avec la dépouille. Le jour des funérailles et les septième, vingt et unième et quarante-neuvième jours de commémoration de mort, la famille en deuil se « blanchit les mains » (gar cajlga-) en offrant des « mérites blancs » (cagaan bujan) aux nombreux convives.

La couleur blanche est considérée comme la plus honorifique (Cevel 1959). Elle renvoie à ce qui est bon et pur, qu'il s'agisse du caractère d'un homme, de l'âme, d'une action, d'un objet (Hamayon 1978 : 218-219). Couleur de la fête, du renouveau, de l'abondance, couleur faste, le blanc est, dans le contexte particulier du rituel funéraire, un agent de purification et il incarne la notion d'accumulation des mérites. Le phénomène de blanchiment se retrouve jusque dans le domaine de la cuisine. Il consiste en une technique de préparation de la viande, correspondant à un traitement particulier de la chair et des os, celle de dégraisser. Cette technique, comme les autres actes de blanchiments, semble sous-tendre un enjeu important lié à la purification et aux mérites. 


\section{La conception mongole de l'âme}

\section{l'âme et les os}

Si l'os est déterminant dans le domaine de la cuisine - il confère un caractère distinctif au plat -, il l'est également dans le domaine de la parenté: le terme « os » (jas) désigne la filiation patrilinéaire ainsi que l'origine, et les expressions où il intervient signifient « naître » ou « mourir » ${ }^{28}$. Selon d'anciennes conceptions chamaniques prébouddhiques, les Mongols croient que leur âme (süns) est logée dans les os (jas) dès la naissance, qu'elle s'y fixe à partir de la petite enfance et, qu'après la mort, elle quitte le corps et se dissocie en trois entités: une âme ou entité reste près de la maison pendant la durée du deuil, une autre âme reste près de la tombe, et une âme renaît. Les croyances mongoles étant désormais fortement teintées de bouddhisme, la renaissance de l'âme s'effectue, positive en un humain et négative en un animal, sur la base d'un jugement instauré par le clergé bouddhique et fixé au quarante-neuvième jour après la mort $^{29}$. Ce jugement se fonde sur les « mérites » (bujan) et peut entraîner un changement d'espèce - on est notamment menacé de renaître en chien ou en araignée si l'on n'est pas assez méritant de son vivant -, représentant en contexte chamanique la perte d'une unité de vie humaine.

Le traitement sacrificiel de la viande (chair-os) ${ }^{30}$ des animaux domestiques et celui, funéraire, du corps des humains (chair-os), étaient auparavant corrélés dans les pratiques et les représentations. Les os de l'animal sacrifié n'étaient pas brisés, mais brûlés au feu, pour que l'âme de l'animal soit offerte au mort au lieu de renaître dans un animal sur terre (Plan Carpin 1965 : 37, 41). Inversement, les os du mort devaient se briser, pour permettre à l'âme de renaître dans un humain, un descendant de même lignée patrilinéaire pour la perpétuer ${ }^{31}$.

Dans les pratiques funéraires contemporaines, l'os n'est plus à l'intersection de la cuisine et de la parenté. L'os de l'animal, dans le traitement duquel résidait le sort de l'âme du défunt, est aujourd'hui exclu du repas funéraire et les techniques de découpe de la chair intriguent. C'est dans le changement de la conception mongole de la renaissance de l'âme humaine que le passage d'un repas de parts de viande à un repas de morceaux « mérites » trouve une explication.

\section{À âme blanchie, viande blanchie}

De la même manière que les humains accumulent de leur vivant des bonnes actions en vue de la bonne renaissance de leur âme à leur mort, une famille en deuil « accumule » (huraa-) des bonnes nourritures pour favoriser le bon sort de l'âme de son défunt parent, c'est-à-dire la « blanchir». Pendant la période de deuil de quarante-neuf jours, par intervalles de sept jours à 
partir des funérailles idéalement fixées au troisième jour suivant la mort, elle accumule et offre des mérites. Le repas des « dix mérites blancs» (arvan cagaan bujan) clôt le rituel funéraire. Consacré aux enfants, qui représentent par analogie les nouveau-nés dans lesquels les âmes des morts renaissent, il se compose de thé au lait, de bouillie de lait, riz et raisins secs, d'une soupe de thé au lait, millet et petits raviolis bouillis, d'une bouillie blanche crémeuse, de bonbons, de petits fromages séchés. Les enfants rapportent chez eux des gâteaux en forme de semelle ${ }^{32}$, des morceaux de sucre et des petites portions de beurre blanc. Ce repas blanc, doux et sucré, de fermeture de deuil répond en quelque sorte au festin d'ouverture de deuil dit " pour déposer l'amer » (gašuu tajlah budaalga) (Ar'jaasüren \& Njambuu 1992 : 365). La période de deuil, amère, sombre, noire, est « supprimée » (dara-) par le blanc, par des mérites blancs et sucrés ${ }^{33}$, en sorte que la matérialité déployée agisse efficacement sur l'aspect immatériel de la mort, l'âme du défunt.

Au-delà des techniques, la cuisine nous parle de l'âme et de sa renaissance. La non-viande de la soupe des funérailles est une viande blanchie et l'on dit également que l'âme du mort « devient une âme blanche ». Ainsi, la soupe funéraire respecte l'interdiction bouddhique de consommation de viande et réunit les conditions nécessaires à une bonne renaissance, bouddhique comme chamanique, de l'âme dans un humain.

\section{$\&$}

Il nous a fallu étudier la conception mongole du repas, remonter jusqu'aux chaînes opératoires de préparation, de distribution et de consommation de la soupe et les comparer à celles de la soupe des funérailles, pour faire la lumière sur les croyances relatives au sort post mortem de l'âme humaine.

C'est finalement le partage d'un repas, égalitaire, sans aucune part distinctive, qui demeure et traverse les siècles. Si l'on se focalise sur l'aliment habituellement discriminant, le partage de la viande perdure: auparavant, des parts indifférenciées provenant de la part (hešig) porteuse de la grâce des ancêtres et rapportées par les sacrifiants; aujourd'hui, des morceaux (heseg), garants de la bonne renaissance de l'âme du mort, consommés par les convives et rapportés par les enfants. Notre analyse rejoint donc celle de Roberte Hamayon qui constate que, chez les Bouriates, le partage égalitaire en parts rapportées était l'aspect fondamental du sacrifice animal (1990 : 786 n. 20).

Les pratiques funéraires des Mongols ont subi des bouleversements importants, mais il semble que l'essentiel des anciennes pratiques chamaniques soit préservé: le nourrissement du mort évoqué par les mérites et le processus d'accumulation des nourritures. Le lieu et la manière de 
procéder ont cependant changé: le mort n'est plus nourri sur le lieu d'enterrement, mais sur l'autel funéraire, dans la yourte où le repas funéraire est désormais consommé. Les prémices de toutes les nourritures du repas des funérailles, à l'exception de la soupe, sont présentées devant le portrait photographique du défunt, sur l'autel funéraire, pour nourrir le mort. Seuls les humains vivants semblent consommer de la soupe, et les boulettes rôties qui la composent. Mais ne serait-ce justement pas une manière détournée de nourrir le mort et indirectement les ancêtres de viande rôtie, comme c'était le cas autrefois? La technique de dégraissage et le mode de cuisson rôti qui détonnaient au départ se justifient et dévoilent ici tout leur sens. Peu importe que le repas comporte de la viande ou non, ou une non-viande, c'est l'acte de nourrissement de l'âme du mort qui compte - acte rituel reposant sur une technique culinaire, celle du rôti.

Il se dégage de notre analyse que les familles mongoles élaborent des tactiques, réajustent leurs pratiques funéraires pour les accorder à la fois avec les lois laïques et hygiénistes du gouvernement communiste et avec les règles religieuses du clergé bouddhique et d'anciennes croyances chamaniques prébouddhiques aujourd'hui renouvelées et désormais tolérées par les pouvoirs politiques et religieux en place.

Une maîtresse de foyer surveillant le feu

Hentij, février 2000.

(Fig. 19)

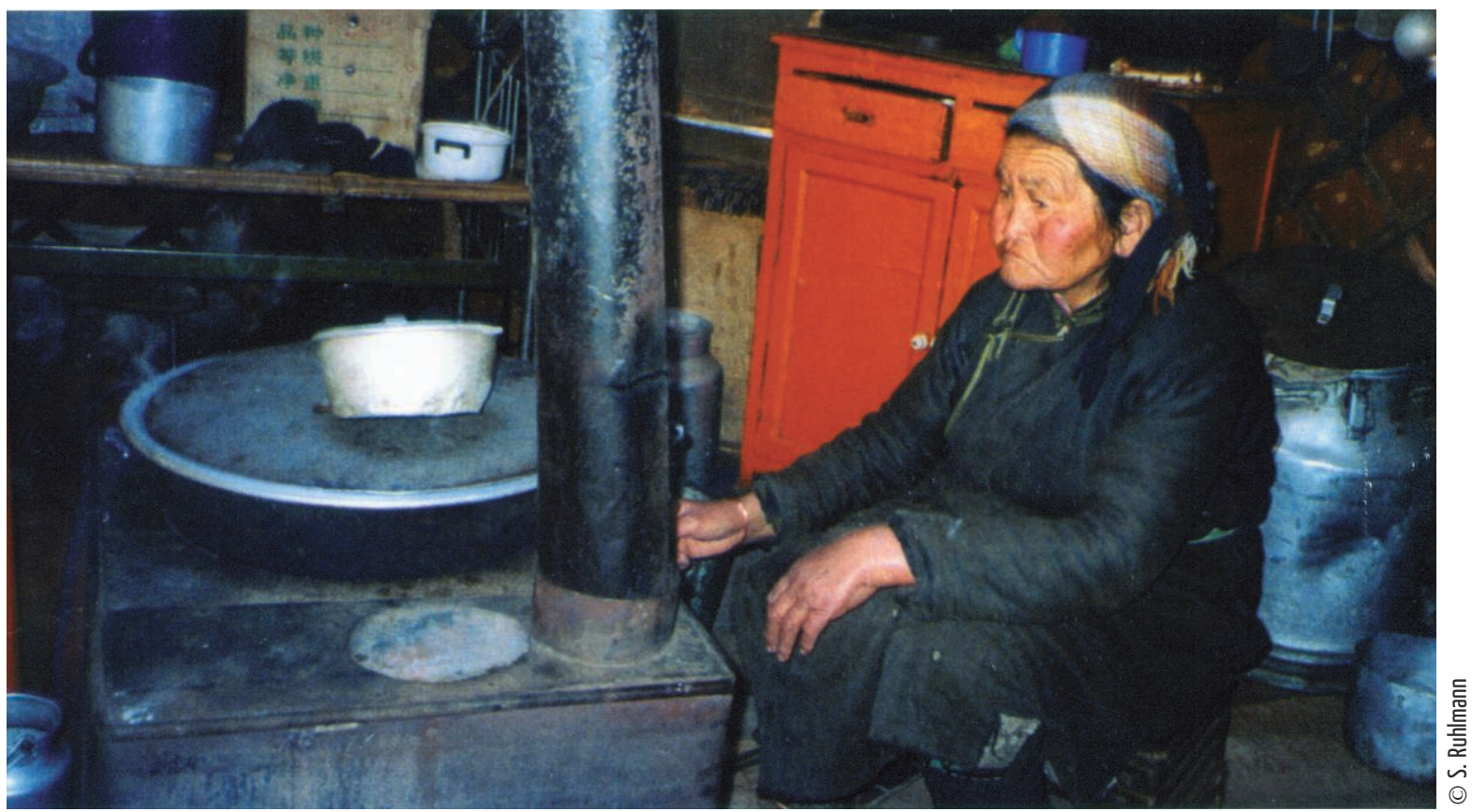


1. Le pays est majoritairement peuplé de Mongols halhs.

2. C'est dans la ville d'Öndörhaan, capitale provinciale du Hentij, région du Nord-Est de la Mongolie, que j'ai observé un rituel funéraire, dans la totalité de son déploiement de quarante-neuf jours. La défunte vivait avec son mari sous une yourte, située dans la même cour d'habitation que leurs deux filles, vivant chacune avec mari et enfants dans une maison en dur. Le veuf était l'oncle paternel aîné du maître du foyer où je résidais et sa femme était tenue de prêter main-forte, comme toutes les parentes proches et éloignées de la défunte et du veuf, pour préparer le repas des funérailles. Concrètement, elle gérait, avec les filles de la défunte, l'organisation du rituel funéraire. Elle m’a demandé de me substituer à sa fille, retenue à Ulaanbaatar, dans sa tâche d'entraide auprès de sa belle-famille en échange de quoi, en ethnologue, j’ai assisté et participé à toutes les étapes du rituel funéraire.

3. Dans l'Histoire secrète des Mongols (Even \& Pop 1994 : 312 notes 63), le terme sölen (šöl sous sa forme halh moderne) qui désigne la soupe de viande ou le bouillon, désigne par euphémisme l’animal destiné à être abattu pour la consommation.

4. L'Histoire secrète des Mongols (Even \& Pop 1994) témoigne de l'importance de la consommation de la viande au XIII siècle.

5. Sur les étapes de fixation de l'âme dans les os pendant la petite enfance et la corrélation avec l'alimentation carnée, détachée de l'os, puis rattachée à l'os, voir Ruhlmann 2009b.

6. La couleur noire désigne à la fois les aliments de qualité inférieure ou qui ne sont pas prêts à être intégrés dans une préparation culinaire et ceux intégrés dans une préparation culinaire mais présentés et consommés sans mélange ni ajout d'autres aliments (Hamayon 1978).

7. Le couteau, dont la pointe est considérée comme une agression envers l'hôte, n'est utilisé au moment de la consommation que lorsqu'il est indispensable, soit pour manger de la viande sur l'os.

8. Pour les techniques de fabrication des pâtes de farine de blé, Ruhlmann 2006 : 143.

9. Les parts constitutives du šüüs préparé chez les Mongols ordos de Mongolie-Intérieure au début du XXe siècle (Mostaert 1968: 638ab) diffèrent peu de celles du šüüs que les Mongols halhs consomment de nos jours (Ruhlmann $2006: 256-272$ ).

10. Quand la tête ne fait pas partie du šüüs, la part la plus honorifique est le sacrum avec la queue, soit la part la plus grasse du mouton.

11. «Plus les pâtes sont fines, plus elles sont belles », répétaient inlassablement les femmes. Une jeune femme mangeait les spaghettis crus qu'elle jugeait avoir coupés trop gros, alors que les Mongols ont une aversion pour le cru - les plus traditionalistes comparent les personnes qui consomment des crudités et des fruits à des moutons broutant de l'herbe, celles qui consomment des farineux à des cochons.

12. La majorité des habitations en milieu urbain, y compris les yourtes, ont l'électricité courante.

13. Nous différencions une chair dégraissée, suivant des étapes techniques précises, d'une chair maigre de nature comme celle de la chèvre.

14. Tandis que le veuf voit sa sociabilité poussée à l'extrême: il reçoit beaucoup de visites et il offre une multitude de nourritures.

15. Pour plus de détails sur les rites d'inversion, Ruhlmann 2006: 458-470 et 2009a.

16. Les éleveurs sacrifient les animaux domestiques qu'ils élèvent, même si par ailleurs ils chassent du gibier. En contexte chamanique, les Mongols sacrifiaient aux âmes des ancêtres le bétail à « museau chaud » (Y. Rintchen 1977), soit des chevaux, en Sibérie, et des moutons, en Mongolie. Chez les Bouriates, le cheval est l'animal sacrificiel des sacrifices collectifs, les moutons des sacrifices privés (Hamayon 1990 : 637-638)

17. Nous connaissons les équivalents halhs modernes des termes: bülüür « batte à beurre » pour bile ür et sarhad « eau-de-vie (de babeurre) » pour sarqud. Nous sommes cependant étonnés que le terme désignant aujourd'hui la batte, un instrument intervenant dans la fabrication du beurre et du lait de jument fermenté, ait pu désigner auparavant les « restes de viande sacrifiée ». Il n'est pas impossible, qu'autrefois, tout rituel reposant sur des libations de produits laitiers ait consisté à partager de la viande. Selon une communication personnelle de Roberte Hamayon, nous pourrions sans doute retracer les changements de sens des verbes partager (de la viande) et asperger (du lait). Ces changements résulteraient du passage d'une économie fondée sur la chasse (même si on élevait aussi des animaux domestiques) à une économie centrée sur l'élevage (même si on y chasse aussi).

18. Les parts de viande détachées des os, parce qu’elles constituent des morceaux de chair indifférenciés, sont égalitaires. Par contre, les parts de viande rattachées à l'os, qui se distinguent suivant l'importance 
de l'os - sa position basse ou haute dans le squelette et sa disposition de dessus ou dessous dans le plat -, constituent des parts hiérarchiques, distribuées aux convives suivant leur degré de parenté et leur statut social (Hamayon 1975).

19. Les losanges de peau, découpés de la tête d'un mouton, occasionnellement offerts au feu du foyer domestique en sont peut-être aussi une illustration.

20. Mostaert 1968 : 149b, 374ab, 414b, 415a, 525b, $686 a$.

21. Grand code de lois, mis en place par l'empereur Gengis.

22. Le bouddhisme tibétain de la branche des Gelugpa s'établit en Mongolie en imposant au peuple mongol une doctrine bouddhique mâtinée d'éléments issus des croyances populaires tibétaines, proches des croyances chamaniques populaires mongoles.

23. Les offrandes d'humains sacrifiés sur les tombes furent également interdites - on offrait à l'âme de l'empereur quarante filles des seigneurs l'ayant servi, Even 1999 : 169. Comme Walter Heissig et Giuseppe Tucci (1973: $376,387)$, nous distinguons les « dons funéraires » de femmes, d'esclaves et de bétail vivants, des sacrifices d'animaux annuels, mensuels ou occasionnels.

24. Le bétail est dès lors devenu une monnaie pour payer dette, amende, dot et pour et pour payer les services religieux des lamas

25. À l'époque mandchoue, seuls les corps des empereurs étaient placés dans des cercueils en bois. Les corps des lamas de haut rang étaient incinérés, quant aux corps des hommes du commun, ils étaient déposés dans la steppe, à même le sol (Ruhlmann 2009a).

26. Pour une description détaillée de la cérémonie d'enterrement, voir Ruhlmann 2006 : 394-470.

27. Aux couleurs (blanc, noir, bleu, vert, jaune, rouge) correspondent des qualités, humaines ou autres, un trait de caractère, une symbolique (Hamayon 1978).

28. Les os de la mère « [se] disloquent » (jas haraca-) pour permettre l'accouchement, ceux du mort sont « déposés » (jas tavi-), expression traduisant à l'origine le dépôt du corps dans la steppe et signifiant aujourd'hui le transport du cercueil au cimetière.

29. Erlig, souverain des Enfers dans le bouddhisme, juge les actions du défunt et fixe le sort de son âme (Even 1999 : 181).

30. Il est à noter que le même terme mah « viande » désigne la chair, animale et humaine.

31. Dans le domaine de la parenté, les os, représentant la parenté du côté du père, sont durables et préservés intacts, tandis que la chair, représentant la parenté du côté de la mère, est périssable et éphémère (Hamayon 1975 : 11).

32. Sur les techniques de fabrication et la symbolique des gâteaux-semelle, Ruhlmann 2008.

33. Chez les Mongols Alashan de Mongolie-Intérieure, le repas de fermeture du deuil est littéralement appelé « supprimer le noir » (hara darah), (Narsu \& Stuart 1994: 99-103). 


\section{RÉFÉRENCES}

Ar'jaasüren, C. \& Njambuu, H., 1992, Grand dictionnaire des coutumes mongoles (Mongol jos zanšlyn ih tajlbar tol'). Ulaanbaatar.

Cevel, JA., 1959, "Les couleurs préférées des Mongols » (" Mongol uudyn erhemledeg öngö »), Studia ethnographica 1(6): 3-5.

Curtin, J., 1909, A Journey in Southern Siberia: the Mongols, their Religion and their Myths. London, Sampson, Low, Martson \& Compagny.

Even, Marie-Dominique \& Pop, Rodica, 1994, Histoire secrète des Mongols. Chronique mongole du XIII siècle. Paris, Gallimard.

Even, Marie-Dominique, 1999, «L'au-delà dans les représentations religieuses mongoles », in Paul Servais éd., La Mort et l'au-delà, Rencontres Orient-Occident. Louvain-la-Neuve, Bruylant Acadmia: 149-196.

Hamayon, Roberte, 1975, « L'os distinctif et la chair indifférente », Études mongoles 6: 99-122.

Hamayon, Roberte, 1978, «Des fards, des mœurs et des couleurs. Étude d'ethno-linguistique mongole », in Tornay S. éd., Voir et nommer les couleurs. Labethno Nanterre: 207-247.

Hamayon, Roberte, 1990, La chasse à l'âme. Esquisse d'une théorie du chamanisme sibérien. Nanterre, Société d'ethnologie.

Heissig, Walter \& Tucci, Giuseppe, 1973, Les Religions du Tibet et de la Mongolie. Paris, Payot.

Humphrey, Caroline, 1999, « Rituals of Death in Mongolia: their Implications for Understanding the Mutual Constitution of Persons and Objects and Certain Concepts of Property », Inner asia 1(1) : 5986.

Lévi-Strauss, Claude, 1965, «Le triangle culinaire », l'Arc 26: 19-29.

Lévi-Strauss, Claude, 1967, L’Origine des manières de table. Mythologiques III. Paris, Plon.

Ligeti, Louis, 1973, "Le sacrifice offert aux ancêtres dans l'Histoire secrète », Acta Orientalia Academiae Scientiarum Hungaricae XXVII (2): 145-161.

Mahias, Marie-Claude, 1985, Délivrance et convivialités. Le système culinaire des Jaina. Paris, Éditions de la Maison des Sciences de l'Homme.

Majnagasev, S.D., 1916, Zertvoprinosenie Nebu u bel'tirov. Sbornik Muzeja antropologii i êtnografii 3: 93 102.

Mintz, Sidney W., 1985, « Core and Fringe », Indian International Centre Quarterly 12 (2) : 193-204.

Mintz, Sidney W. \& Schlettwein-Gsell, Daniela, 2001, « Food Patterns in Agrarian Societies: the "CoreFringe-Legume Hypothèses " a Dialogue », Gastronomica I (1) : 41-52.

Mostaert, Antoine, 1968 [1941], Le Dictionnaire Ordos. Londres, CICM.

Narsu \& Stuart, Kevin, 1994, «Funerals in Alxa right banner, Inner Mongolia ». Mongolian studies, Journal of the Mongolian Society XVII : 99-103.

Plan Carpin, Jean de, 1965, Histoire des Mongols. J. Becquet et L. Hambis (trad.), Paris, AdrieuMaisonneuve.

Radlov, W., 1893, Aus Siberien. Lose Blätter aus meinem Tagenbuche. Leipzig, T.O. Weigel.

Riazanovski, V. A., 1965 [1937], Fundamental Principles of Mongol Law. Uralic and Altaic Series, vol.43, Indiana University Publications.

Rintchen, Y., " Pourquoi on offre des chevaux et des moutons aux esprits chamaniques Mongols », L'Ethnographie 74-75: 155-156.

Ruhlmann, Sandrine, 2006, « Le partage des prémices et du fond de la marmite. Essai d'anthropologie des pratiques alimentaires chez les Mongols Xalx », Thèse de doctorat, EHESS, 574p.

Ruhlmann, Sandrine, 2008, « Une curieuse pâtisserie en forme de semelle: le nouveau support de bonheur des Mongols halhs bouddhisés », Anthropology of Food, Varia, http://aof.revues.org.

Ruhlmann, Sandrine, 2009a, «L'enterrement chez les Mongols contemporains: le cercueil, la tombe et la yourte miniature du mort ». Études mongoles \& sibériennes, centrasiatiques \& tibétaines 40, à paraître sur le site http://emscat.revues.org/. 
Ruhlmann, Sandrine, 2009b, « Le bouillon de naissance de l'accouchée chez les Mongols Xalx: fermer son corps pour s'ouvrir socialement », Monumenta Serica, Festschrift pour F. Aubin, sous la dir. de D. Aigle, I. Charleux, V. Goossaert et R. Hamayon, à paraître.

Tomka, Paul, 1965, «Les termes de l'enterrement chez les peuples mongols ». Acta Orientalia Hungarica XVIII(1-2) : 159-181.

Vassas, Claudine, 2001, «En Roumanie, l'autre moitié du rite: les cuisinières des morts », Clio. Histoire, Femmes et Société 14: 93-118.

Yalman, Nur, 1971, « On the Meaning of Food Offerings in Ceylon », in Proceedings of the 1969 Annual Spring Meeting of the American Ethnological Society. Seattle-London, University of Washington Press: 81-96.

\section{RÉSUMÉ}

Une soupe peu ordinaire. Aussi loin que l'on remonte dans les sources historiques, les Mongols apparaissent comme étant des mangeurs de viande. Au XIII siècle, ils consommaient la viande accompagnée d'un bol de son bouillon de cuisson. Aujourd'hui, chez les Mongols de la République de Mongolie, composée majoritairement de Mongols halhs, l'élément principal du repas n'est autre qu'une soupe de viande. Selon la définition mongole, la viande qui nourrit et fait repas est nécessairement fraîche et grasse. Le mode de cuisson qui confère à la viande ses propriétés nutritives et préserve ses qualités requises est le bouilli.

La fin du XVe siècle, date de leur conversion au bouddhisme, marque un tournant dans la vie des Mongols, à commencer par leurs pratiques funéraires: ils se voient interdits de sacrifier des animaux et de consommer de la viande à l'occasion de la mort d'un humain. En réalité, cet interdit bouddhique, qui se traduit par des règles alimentaires, concerne à la base le partage de la part sacrificielle de viande destinée aux mânes des ancêtres, cette part qui devait être rôtie pour leur parvenir.

Le repas des funérailles comporte cependant, aujourd'hui, de la viande. À partir d’une étude des chaînes opératoires de préparation, de distribution et de consommation de la viande, l'auteur montre comment une série d'inversions, que justifie la mort d'un humain, se retrouve jusque dans les techniques de préparation, de distribution et de consommation de la viande de la soupe offerte au repas des funérailles - soupe revêtant une forme toute particulière.

À partir d'un cas particulier observé, l'auteur retrace, sur la base d'une étude des techniques culinaires, les évolutions des pratiques funéraires mongoles et les croyances qui leur sont attachées. Les croyances populaires chamaniques prébouddhiques et bouddhiques, relatives à l'âme et à son devenir post mortem, n'ont pas été complètement éradiquées, ni par trois siècles de politique anti-chamanique du clergé bouddhique ni par un demi-siècle de politique antireligieuse du parti politique communiste.

Les Mongols consomment une viande non ordinaire - fraîche mais techniquement dégraissée et rôtie -, et semblent s'en accommoder en contexte funéraire, tandis qu'ils seraient offensés de se la voir offrir et de devoir la manger au quotidien. Ne serait-ce pas là une manière de nourrir l'âme du mort d'une viande rôtie comme la pratique chamanique prébouddhique le préconisait au XIII siècle à travers le sacrifice animal?

\section{ABSTRACT}

An Unordinary Soup. As far as we go back to historical sources, Mongolian people appear to be meat eaters. At the thirteenth century, they were consuming the meat served with its cooking bouillon. Today, in the Republic of Mongolia, composed of a majority of Halh, the main element of the meal is none other than a meat soup. According to the Mongolian definition, the meat that nourishes and makes meal is necessarily fresh and fat. The cooking mode that confers to the meat its nutritive properties and preserves its required qualities is the boiled mode. 
The end of the fifteenth century, date of the conversion to Buddhism, marks a turning point in the life of the Mongols, beginning with their funerary practices: the sacrifice of animals has been prohibited as much as the consumption of meat at the death of a human. In fact, this Buddhist prohibition by food rules specifically the sharing of the sacrificial meat offered to ancestors' manes, this part that was to be roasted to reach ancestors.

However, today the funerary meal contains meat. From the study of the operating chains of preparation, distribution and consumption of meat, the author shows how a series of inversions, that humans' death justifies, is found even in the techniques of preparation, distribution and consumption of the meat of the soup offered at the funerary meal - soup assuming a specific form.

From the observation of a particular case, the author retraces, on the basis of a culinary techniques' study, changes in Mongolian funerary practices and beliefs that are connected. The prebuddhist shamanistic popular beliefs and the Buddhist beliefs concerning the soul and its post-mortem fate have not been completely eradicated, neither by three centuries of anti Shamanistic policy of the Buddhist clergy nor by a half-century of antireligious policy of the Communist Party. In funerary context, the Mongols eat a non-ordinary meat -fresh but technically fat skimmed and roasted-and seem to put up with, whereas they would be offended to receive and have to eat it on a daily basis. Isn't there a way to nourish the soul of the dead with roasted meat such as the prebuddhist shamanistic practice recommended it at the thirteenth century through the animal sacrifice?

\section{MOTS CLÉS}

Pratique alimentaire, alimentation, soupe, viande, funérailles, mort, chamanisme, bouddhisme, âme, mérites.

\section{KEYWORDS}

Food practice, alimentation, soup, meat, funeral, death, Shamanism, Buddhism, soul, merits. 